\title{
Calcium Signaling Pathways Mediating Synaptic Potentiation Triggered by Amyotrophic Lateral Sclerosis IgG in Motor Nerve Terminals
}

\author{
Mario R. Pagani, ${ }^{1}$ Ricardo C. Reisin, ${ }^{2}$ and Osvaldo D. Uchitel ${ }^{1}$ \\ ${ }^{1}$ Laboratorio de Fisiología y Biología Molecular, Instituto de Fisiología Biología Molecular y Neurociencias UBA-CONICET, Facultad de Ciencias Exactas y \\ Naturales, Universidad de Buenos Aires, Ciudad Universitaria, C1428EHA Buenos Aires, Argentina, and 2Servicio de Neurofisiología, Hospital Británico, \\ C1280AEB Buenos Aires, Argentina
}

\begin{abstract}
Sporadic amyotrophic lateral sclerosis (ALS) is a neurodegenerative disease that affects particularly motoneurons. Several pieces of evidence suggested the involvement of autoimmune mechanisms mediated by antibodies in ALS. However, the significance of those antibodies in the disease and the underlying mechanisms are unknown. Here we showed that IgG purified from a group of sporadic ALS patients, but not familial ALS patients, specifically interact with the presynaptic membrane of motoneurons through an antigen-antibody interaction and modulated synaptic transmission. Immunoreactivity against nerve terminals showed strong correlation with synaptic modulation ability. In addition, several controls have ruled out the possibility for this synaptic modulation to be mediated through proteases or nonspecific effects. Effective IgG potentiated both spontaneous and asynchronous transmitter release. Application of pharmacological inhibitors suggested that activation of this increased release required a nonconstitutive $\mathrm{Ca}^{2+}$ influx through $\mathrm{N}$-type $\left(\mathrm{Ca}_{\mathrm{v}} 2.2\right)$ channels and phospholipase $\mathrm{C}$ activity and that activation of $\mathrm{IP}_{3}$ and ryanodine receptors were necessary to both activate and sustain the increased release. Consistent with the notion that ALS is heterogeneous disorder, our results reveal that, in $\sim 50 \%$ of ALS patients, motor nerve terminals constitutes a target for autoimmune response.
\end{abstract}

Key words: calcium channels; phospholipase $\mathrm{C}$; $\mathrm{IP}_{3} \mathrm{R}$; RyR; calcium homeostasis alteration; signaling mechanisms

\section{Introduction}

Sporadic amyotrophic lateral sclerosis (ALS) is the most common adult-onset motoneuron degenerative disease, with an invariably fatal prognosis. A variety of mechanisms have been suggested to explain ALS pathogenesis (Cleveland and Rothstein, 2001; Strong, 2003), and several lines of evidence support the involvement of autoimmune mechanisms. T-cell infiltration at the spinal cord (Troost et al., 1990) and degenerative structural alterations associated with intracellular $\mathrm{Ca}^{2+}\left(\left[\mathrm{Ca}^{2+}\right]_{\mathrm{i}}\right)$ increment in motor nerve terminals have been observed in ALS patients (Siklos et al., 1996). Also, autoantibodies against ganglioside GM1, neurofilament proteins, tumor necrosis factor receptor member FAS (CD95), and voltage-dependent calcium channels (VDCCs) (Smith et al., 1992; Drachman, 2000) have been reported. Interestingly, some of this evidence correlates with observations made in research animals. In rodents treated with IgG from ALS patients (ALS-IgG), a $\left[\mathrm{Ca}^{2+}\right]_{\mathrm{i}}$ increment as-

\footnotetext{
Received June 24, 2005; revised Jan. 20, 2006; accepted Jan. 22, 2006.

This work was supported by Agencia Nacional de Promoción Científica y Tecnológica Grant 6220 and Universidad de Buenos Aires Ciencia y Técnica Grant X171. We thank Licentiate Lorena Rela and Emiliano Merlo, Dr. Fernando Marengo for advice, and Dr. Angela Vincent for ELISAs and critical reading of this manuscript.

Correspondence should be addressed to Osvaldo D. Uchitel, Laboratorio de Fisiología y Biología Molecular, Facultad de Ciencias Exactas y Naturales, Universidad de Buenos Aires, Ciudad Universitaria, Pabellón II-2 ${ }^{\text {do }}$ piso, C1428EHA Buenos Aires, Argentina. E-mail: odu@fbmc.fcen.uba.ar.

D01:10.1523/JNEUROSCI.4394-05.2006

Copyright $\odot 2006$ Society for Neuroscience $\quad$ 0270-6474/06/262661-12\$15.00/0
}

sociated with degenerative structural alterations in motoneurons and synaptic plasticity at neuromuscular transmission was also observed (Engelhardt et al., 1995; Fratantoni et al., 2000; Pullen and Humphreys, 2000; Pullen et al., 2004). ALS-IgG from 50\% of ALS patients trigger synaptic potentiation in spontaneous neuromuscular transmission, together with $\left[\mathrm{Ca}^{2+}\right]_{\mathrm{i}}$ increment $(e x$ vivo), and can induce long-term neuromuscular dysfunction in vivo (Uchitel et al., 1988, 1992; Engelhardt et al., 1995; O'Shaughnessy et al., 1998; Mohamed et al., 2002). These findings suggest that ALS-IgG may be involved in ALS pathogenesis. However, the presence and significance of these autoantibodies are still under discussion (Drachman, 2000). Also, therapeutic immunosuppression has been ineffective, but, in all cases, the disease was exceedingly advanced (S. A. Smith et al., 1994). Thus, we focused our investigations on the earliest functional alterations of the disease. We thought that ALS-IgG-mediated synaptic potentiation (ALS-IgG-sp) is the consequence of an increase in $\left[\mathrm{Ca}^{2+}\right]_{\mathrm{i}}$ of the motor nerve terminal (MNT) (Engelhardt et al., 1997). Consequently, deregulation of $\left[\mathrm{Ca}^{2+}\right]_{i}$ may lead to mitochondrial dysfunction, intracellular free radical damage, and cell death (Guegan et al., 2001; Strong, 2003). Therefore, ALS-IgG-sp may be an early step of motoneuron degeneration in ALS. However, the significance of ALS-IgG in the alterations reported is still unclear because of opposite results by different research groups (cf. Smith et al., 1992; Arsac et al., 1996) and because of a report that showed proteolytic activity in IgG fractions from sera of ALS 
patients (Nyormoi, 1996). A key issue is, therefore, to rule out that ALS-IgG-sp is produced by contaminants.

Although neuronal death could be mediated by ALS-IgG, the mechanisms underlying ALS-IgG-sp have not yet been identified. An understanding of ALS-IgG mechanism would provide useful insight not only into the relevance of autoimmune mechanisms in ALS pathogenesis but also into the prevention of motoneuronspecific cell degeneration. To address these questions, we investigated the significance of ALS-IgG in synaptic modulation, the localization of their antigenic target, and the signaling mechanisms underlying ALS-IgG-sp.

\section{Materials and Methods}

\section{Healthy controls and ALS patients}

Sera were obtained from 13 sporadic ALS patients (age, 42-68 years; eight males and five females) and 15 control patients (age, 41-70 years; eight males and seven females). We used antibodies from two types of control patients: healthy control (h-Ctrl-IgG) $(n=6)$ and disease control $(\mathrm{d}-\mathrm{Ctrl}-\mathrm{IgG})(n=9)$. Individual antibodies are identified with numbers from 1 to 13 (for ALS), capitals letters from A to F (for healthy controls), and lowercase letters from a to i (for disease controls). Disease control patients had been diagnosed as familial ALS (fALS) ( $n=3 ; \mathrm{a}, \mathrm{b}$, and c), Alzheimer's disease $(n=1 ; \mathrm{d})$, myasthenic syndrome $(n=2$; e and $\mathrm{f})$, peripheral neuropathy $(n=2$; g and $\mathrm{h})$, and Charcot-Marie-Tooth disease $(n=1 ; \mathrm{i})$. The h-Ctrl-IgG and d-Ctrl-IgG jointly will be named Ctrl-IgG. ALS patients had been diagnosed as definite ALS according to the El Escorial criteria (1994).

\section{IgG purification}

Proteins from sera were precipitated by ammonium sulfate and dialyzed against $20 \mathrm{~mm}$ phosphate buffer. IgG were purified by affinity chromatography using a HiTrap protein G HP column (Amersham Bioscience, Piscataway, NJ) according to the recommendations of the supplier. IgG were dialyzed against $10 \mathrm{~mm}$ PBS using a $12 \mathrm{kDa}$ pore membrane (Millipore, Bedford, MA) membrane filtration on a $2 \mu \mathrm{m}$ filter (Millipore), and addition of BSA was performed before storage in small volumes at $-20^{\circ} \mathrm{C}$. Several fractions of each IgG were used to estimate IgG concentration, using a spectrophotometer reading optical densities at $\lambda_{280 \mathrm{~nm}}$ and correcting for human IgG. A 10\% SDS-PAGE was used to test the purified IgG. Human sera were decomplemented by heating at $56^{\circ} \mathrm{C}$ for $30 \mathrm{~min}$ and dialyzed against normal solution before use.

Anti-GM1 ganglioside antibodies were not detected in the IgG preparations using a standard ELISA at IgG concentrations of $0.05 \mathrm{mg} / \mathrm{ml}$ (Prof. Angela Vincent, Department of Clinical Immunology, Oxford Radcliffe Hospital Trust, Oxford, UK).

\section{Animals and muscle preparation}

Animals were cared for in accordance with national guidelines for the human treatment of laboratory animals, which are comparable with those established by the National Institutes of Health. All efforts were made to minimize animal suffering and the number of animals used.

Experiments were performed on diaphragm, soleus, and levator auris muscle from male Swiss mice weighing 25-30 g. Mice were anesthetized with $2 \%$ tribromoethanol and exsanguinated, after which muscles were removed and dissected on a Sylgard-coated dish (Dow Corning, Midland, MI). Tissue was kept covered with a Ringer's normal solution (RN) [in mM: $137 \mathrm{NaCl}, 5 \mathrm{KCl}, 2 \mathrm{CaCl}_{2}, 1 \mathrm{MgSO}_{4}, 12 \mathrm{NaHCO}_{3}, 1 \mathrm{Na}_{2} \mathrm{HPO}_{4}$, and 11 glucose (continuously bubbled with $95 \% \mathrm{O}_{2}-5 \% \mathrm{CO}_{2}$ )] and then transferred to a recording chamber to perform electrophysiological experiments at $25^{\circ} \mathrm{C}$ or was used for immunohistochemical analysis.

\section{Electrophysiology}

To evaluate spontaneous and evoked synaptic activity, miniature endplate potentials (MEPPs) and evoked endplate potentials (EEPs) were measured by intracellular recording. The electrophysiological equipments used have been described previously (Fratantoni et al., 2000). Borosilicate glass capillaries were used for microelectrodes filled with $3 \mathrm{M}$ $\mathrm{KCl}$ (20-35 M $\Omega$ resistance). Normal synaptic activity was confirmed in $\mathrm{RN}$ for all neuromuscular preparations used, after which IgG or drugs were evaluated. To perform the largest number of experiments with the IgG from each patient, we first found out the effective working concentration in each IgG sample $(100 \mu \mathrm{g}$ to $400 \mu \mathrm{g} / \mathrm{ml})$. Because ALS-IgG incubating time was necessarily $\sim 4 \mathrm{~h}$, incubations were performed into a wet incubation chamber using $\mathrm{RN}$ so as to keep constant chamber humidity and consequently constant volume, viscosity, ion concentration, and $\mathrm{pH}$ of the incubation solution. Thus, all experiments shown here were made with $4 \mathrm{~h}$ incubation, except when stated. Control experiments were performed parallel to experiments in which IgG and/or drugs were used to evaluate synaptic activity, to rule out possible false-positive or false-negative results. Diaphragm muscles were removed, and, alternatively, the left or right hemidiaphragm was used to study the effects of ALS-IgG or Ctrl-IgG. All of the experiments performed with photosensitive drugs were performed avoiding direct illumination on the preparations. Goat IgG anti-human and goat $\operatorname{IgG}$ anti-rabbit were used for ALS-IgG or Ctrl-IgG immunoprecipitation (IP). Loading of BAPTA-AM was made in $0 \mathrm{mM} \mathrm{Ca}^{2+}$ and $3 \mathrm{mM} \mathrm{Mg}^{2+}$ for $30 \mathrm{~min}$ at $25^{\circ} \mathrm{C}$, and for 30 min at $37^{\circ} \mathrm{C}$, and then rinsing at $25^{\circ} \mathrm{C}$ in $0 \mathrm{Ca}^{2+}$ and $\mathrm{RN}$.

At least 30 synapses from three different animals were examined in each condition. Values are expressed as mean \pm SEM. Statistical significance was evaluated by a one-tailed Student's $t$ test by comparing the mean synaptic activity with their own control in normal solution before any treatment with antibodies or drugs, except when stated.

Amplitudes of MEPPs at each synapse were normalized to $-75 \mathrm{mV}$ : $A_{\text {Nor }}=A \times\left(-75-E_{\mathrm{r}}\right) / E_{\mathrm{m}}$, where $A$ is the amplitude measured in millivolts, $E_{\mathrm{r}}$ is the reversal potential [acetylcholine receptor $(\mathrm{AChR})$ is assumed to be $0 \mathrm{mV}$ ], and $E_{\mathrm{m}}$ is the mean membrane potential during the recording period. Interevent intervals measured were plotted and fitted to a one-order exponential and analyzed by $\chi^{2}$ test (Origin 6.0; Microcal Software, Northampton, MA). All measurements on the recordings were performed using Clampfit 9.0 (Molecular Devices, Palo Alto, CA).

To evoke EPPs, the phrenic nerve was stimulated with two platinum electrodes coupled to a stimulus isolation unit (Grass Stimulator; Grass Instruments, Quincy, MA). $\mu$-Conotoxin GIIIB at $1 \mu \mathrm{M}$ (Alomone Labs, Jerusalem, Israel) was used to prevent muscle contraction. Evoked neurotransmitter release was evaluated at a low $\left[\mathrm{Ca}^{2+}\right] /\left[\mathrm{Mg}^{2+}\right]$ ratio, in which quantal content (QC) was estimated by the coefficient of variation. QC at 20 and $40 \mathrm{~Hz}$ was estimated after $6 \mathrm{~s}$ stimulation to avoid the initial facilitation and depression in a train of stimuli.

Paired-pulse facilitation (PPF) and posttetanic potentiation (PTP) were studied in the low $\left[\mathrm{Ca}^{2+}\right] /\left[\mathrm{Mg}^{2+}\right]$ solution $\left(2 \mathrm{mM} \mathrm{Ca}^{2+} / 6.5 \mathrm{mM} \mathrm{Mg}^{2+}\right)$. PPF was evaluated by the change in EPP amplitude in the second EPP normalized to the first one. PTP was evaluated by comparing 10 stimuli at $0.5 \mathrm{~Hz}$ before and after a tetanic stimulation (500 stimuli at $100 \mathrm{~Hz}$ ).

\section{Tissue and immunohistochemistry}

Immunohistochemistry on muscle cross sections. Diaphragm or soleus muscle were removed and fixed without delay for 3 min with $4 \%$ paraformaldehyde (PFA) in $0.1 \mathrm{~m}$ phosphate buffer, $\mathrm{pH} 7$, at room temperature. Then, they were washed in phosphate buffer for $1 \mathrm{~min}$, permeabilized in 1\% Triton X-100 for $5 \mathrm{~min}$, washed again in phosphate buffer for $1 \mathrm{~min}$, and finally cryoprotected in $30 \%$ sucrose. All of the previous steps were done at $4^{\circ} \mathrm{C}$, except when stated. Blocks of tissue were embedded in Tissue-Tek (OCT4583; Sakura, Tokyo, Japan), in a sealed plastic tube, and frozen. Cross sections, $40 \mu \mathrm{m}$ thick, were cut with a cryostat, and muscle sections were mounted onto gelatin-coated slides, air dried for 15 $\min$, and stored at $-20^{\circ} \mathrm{C}$.

Muscle sections were processed simultaneously for double labeling by indirect immunofluorescence, and direct staining was performed with $\alpha$-bungarotoxin coupled to tetramethylrhodamine (BgTx-R) (Invitrogen, Carlsbad, CA). Visualization of the human IgG was through a tagged IgG with FITC and of the anti- synaptotagmin antibody with a tagged IgG with Alexa Fluor 488 (Invitrogen). All incubations were performed at room temperature, using $10 \mathrm{~mm}$ PBS, $\mathrm{pH}$ 7.4, except when stated. Immunofluorescence experiments were performed according to Pagani et al. (2004). In brief, sections were permeabilized with $0.1 \%$ Triton X-100, rinsed in PBS, and incubated with the primary antibody for $19 \mathrm{~h}$ at $4^{\circ} \mathrm{C}$. Tissue was washed with PBS for 90 min. Sections were then incubated simultaneously with the secondary antibody and BgTx-R for $105 \mathrm{~min}$ 
and rinsed for $40 \mathrm{~min}$ in PBS. Finally, sections were mounted using ProLong Antifade (Invitrogen). To define the specific distribution patterns and autofluorescence level, control assays were performed with Ctrl-IgG as primary antibody or without primary antibody. No staining was observed in any control assay.

Immunohistochemistry on whole muscle fiber. Levator auris muscles were removed from the mice and carefully dissected, stretched, fixed with $1 \%$ PFA for $5 \mathrm{~min}$, and rinsed in PBS. All of the procedures were done at $4^{\circ} \mathrm{C}$ using filtered solution through a $50 \mu \mathrm{m}$ pore size (Millipore). The primary antibody $(200 \mu \mathrm{g} / \mathrm{ml}$ ALS-IgG or Ctrl-IgG) was incubated overnight and washed for $20 \mathrm{~min}$. The secondary FITC-labeled antibody (10 $\mu \mathrm{g} / \mathrm{ml}$ ) and BgTx-R were incubated simultaneously for $2 \mathrm{~h}$ and rinsed for $1 \mathrm{~h}$ with PBS.

\section{Microscopy and photography}

A confocal microscope (BX61-FV300; Olympus Optical, Tokyo, Japan) equipped with an argon/helium-neon-G laser that allows simultaneous scanning was used to view and acquire the images of the immunofluorescent sections. Confocal images from cross-sectioned muscles were obtained with a PlanApo oil-immersion lens, $100 \times$ objective, numerical aperture (NA) 1.4; those from whole-muscle fibers were obtained with a LUMplanFl water-immersion objective lens, 60×, NA 0.9 (Olympus Optical). For experimental details, see Pagani et al. (2004). Mean values of fluorescence signal for a particular antibody was obtained by optical density quantification after background subtraction from 12 synapses from three different animals using FluoView 300 (Olympus Optical). Laser alignment was tested by $4.2 \mu \mathrm{m}$ Tetra speck microsphere (Invitrogen).

\section{Chemicals}

Salts and all other reagents used were of analytical grade and purchased from Sigma (St. Louis, MO), except when stated. Tribromoethanol was purchased from Aldrich (Milwaukee, WI). Dihydropyridine was from Research Biochemicals (Natick, MA), and protease inhibitors were from Sigma (aprotinin, pepstatin A, and EGTA) or Calbiochem (San Diego, CA) (aprotinin and pepstatin A). BAPTA-AM and the synthetic polypeptide $\omega$-conotoxin GVIA (GVIA) and $\omega$-agatoxin IVA were from Alomone Labs.

\section{Results \\ ALS-IgG triggers reversible synaptic potentiation of spontaneous transmitter release}

ALS-IgG-mediated synaptic potentiation has been reported previously (Uchitel et al., 1988, 1992). However, there are some uncertainties as to whether a component different from ALS-IgG may be responsible for the structural and functional modifications observed (Nyormoi, 1996). Thus, to evaluate the effect of ALS-IgG on synaptic activity, MEPPs were recorded at neuromuscular junction (NMJ) before and after application of ALSIgG or control antibodies. Neither the Ctrl-IgG (15 of 15) nor $54.5 \%$ of the ALS-IgG ( 6 of 11) had an effect on MEPP frequency (Fig. 1A). However, ALS-IgG purified from the remaining five ALS patients increased synaptic activity after $4 \mathrm{~h}$ of incubation (Fig. $1 A$ ). Synaptic activity was enhanced 2.5 - to 3 -fold compared with the control, without IgG or with control antibodies (Fig. $1 A, B)$. Increment in synaptic activity correlated positively with incubation time, reaching a maximal rate of frequency of MEPP $(759 \pm 211$ per minute) after $9 \mathrm{~h}$ of incubation (Fig. 1C). Positive ALS-IgG were used in the following experiments.

ALS-IgG-sp was independent of action potentials, because it was not affected by the presence of tetrodotoxin $(0.5 \mu \mathrm{g} / \mathrm{ml})$ (Fig. $1 D)$. To test whether the ALS-IgG effect could be attributable to a proteolytic action through a contaminant serine protease $(\mathrm{Ny}-$ ormoi, 1996), we studied the effect of ALS-IgG on spontaneous synaptic activity by using ALS-IgG and muscles preincubated with protease inhibitors. The specific serine protease inhibitor (10 $\mu \mathrm{g} / \mathrm{ml}$ aprotinin) was not able to preclude the ALS-IgG-sp.
Moreover, the serine protease inhibitor alone did not introduce modifications at normal MEPP frequency. Furthermore, we studied aspartic and metalloprotease inhibition using pepstatin A $(10 \mu \mathrm{M})$ and EGTA $(3 \mathrm{mM})$, respectively. Inhibition of these proteases did not prevent ALS-IgG-sp, even when said protease inhibitors were used all together (Fig. 1D). These results indicated that the synaptic potentiation was independent of protease activity (Fig. 1D).

After $6.5 \mathrm{~h}$ of incubation, ALS-IgG-sp was completely reversed by rinsing the nerve muscle preparation for $30-45 \mathrm{~min}$ with normal solution (Fig. 1D), adding support to the idea that ALS-IgG-sp was not the result of an irreversible cellular damage, as could be expected from a contaminating proteolytic activity. This fast reversibility also indicated a weak interaction between the ALS antibodies and their antigenic target.

To further test the requirement of ALS-IgG to induce potentiation of spontaneous transmitter releases, we immunoprecipitated ALS-IgG or Ctrl-IgG using anti-human antibodies or antirabbit antibodies as control. The products of IP were used to evaluate their capability to modulate synaptic transmission. Incubation of the IP of ALS-IgG with anti-human antibodies did not alter transmitter release. In contrast, we observed synaptic potentiation in muscles incubated with the IP of ALS-IgG with anti-rabbit antibodies (Fig. 1D). As expected, Ctrl-IgG immunoprecipitated with anti-human antibodies had no effect on synaptic activity (Fig. 1D). These results showed that synaptic potentiation is ALS-IgG dependent.

To characterize ALS-IgG-sp, we studied whether ALS-IgG changes the properties of spontaneous synaptic activity. We examined MEPP frequency distribution, mean amplitude of MEPPs, and interval time distribution between MEPPs. MEPP frequency distribution was more spread out after ALS-IgG incubation $(p<0.05)$ (Fig. $2 A, B$ ). However, we did not find significant differences in interval time distribution or in the mean amplitude of MEPPs recorded from ALS and control treated muscles $(p>0.05)$ (Fig. $2 A-F)$. We rarely observed giant postsynaptic potentials. Absence of changes in mean amplitude of MEPPs after ALS-IgG application indicated that quantal size was not affected by ALS-IgG (Fig. 2E,F).

\section{ALS-IgG did not affect action potential-evoked synaptic transmission}

Although antibodies from ALS patients affect spontaneous synaptic transmission and $\mathrm{Ca}^{2+}$ current (Uchitel et al., 1988; Delbono et al., 1991), very little is known about the effect of ALS-IgG on action potential-evoked synaptic transmission. O'Shaughnessy et al. (1998) observed an increment in QC mediated by ALS-IgG at low frequency of electrical stimulation. However, studying anconeus muscles from ALS patients, Maselli et al. (1993) detected a reduction in QC with respect to control patients.

Despite the fact that ALS-IgG-sp was independent of action potential (Fig. 1D), ALS-IgG can possibly affect mechanisms common to spontaneous and evoked synaptic transmission (i.e., a particular type of calcium signal). Thus, we investigated the effect of ALS-IgG on EPPs evoked by nerve stimulation at physiological frequencies of 20 and $40 \mathrm{~Hz}$ (Norris and Gasteiger, 1955). We examined the effect of ALS-IgG, which enhanced spontaneous neurotransmission, in which each antibody was studied on at least 30 synapses from three diaphragm muscles. ALS-IgG application did not modify QC of transmitter release. The QC values measured at 20 and $40 \mathrm{~Hz}$ were similar to those obtained from neuromuscular preparations incubated with 


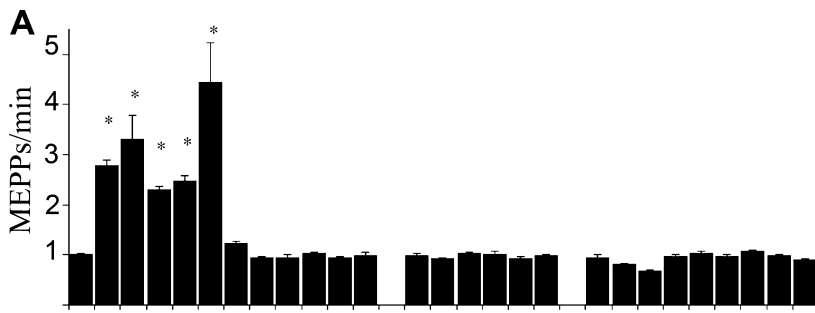

RN $122 \quad 3 \quad 456678891011$ A B C DE F a b c d ef $g h i$

B

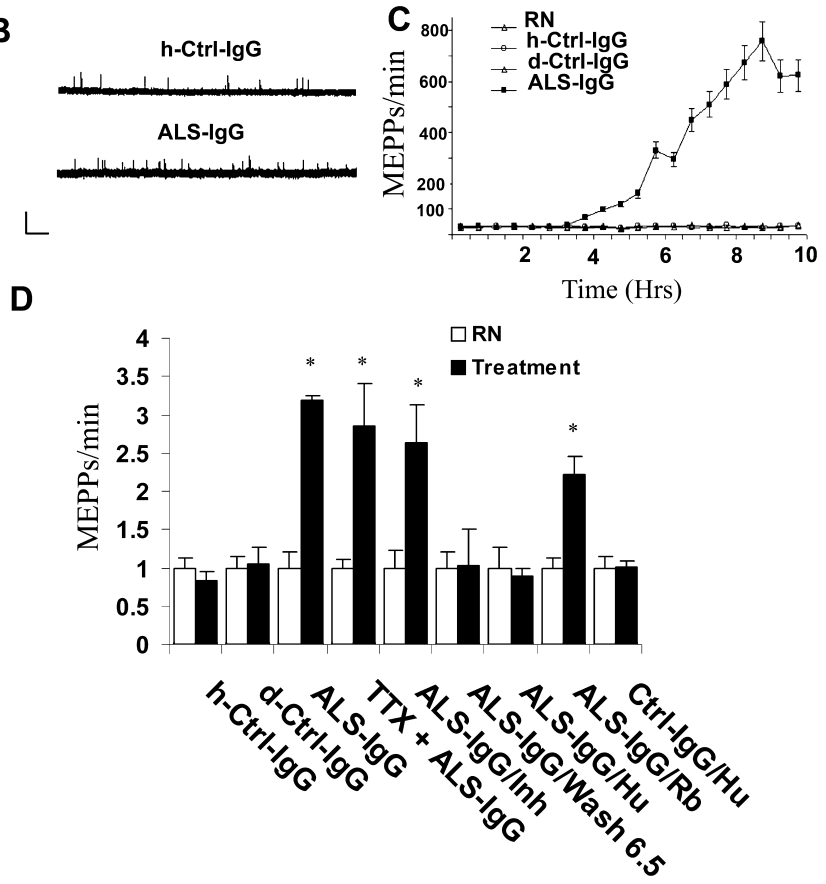

Figure 1. Specific and reversible synaptic potentiation at mouse diaphragm neuromuscular synapses by lgG purified from ALS patients. A, Normalized synaptic transmission (MEPPs per minute) in neuromuscular synapses after $4 \mathrm{~h}$ incubation in normal solution (RN) or with antibodies purified from ALS patients (from 1 to 11), healthy individuals (from A to F), and patients with other diseases (from a to i), as described in Materials and Methods. A range of time of incubation (between 4 and $7 \mathrm{~h}$ ) and antibodies concentration $(100 \mu \mathrm{g} / \mathrm{ml}$ to $4.5 \mathrm{mg} / \mathrm{ml})$ was studied. Note that ALS-lgG from patients $1-5$ increased synaptic activity $2.5-3$ times as much as in control activity. $\boldsymbol{B}$, A sample recording showing that application of human ALS-lgG (100 $\mu \mathrm{g} / \mathrm{ml})$ increases MEPP frequency (bottom) compared with the normal frequency (RN) (top) at room temperature $\left(25^{\circ} \mathrm{C}\right)$. Calibration: $1 \mathrm{mV}$, $1000 \mathrm{~ms}$. C, Absolute mean synaptic activity recorded during several hours after application of ALS-lgG (from patients 1-5), h-Ctrl-IgG (from patients A-C), d-Ctrl-lgG (from patients d, e, and i), or without $\lg G(\mathrm{RN})$. After $4 \mathrm{~h}$ incubation, ALS-lgG trigged synaptic potentiation, which correlated positively with incubation time. Control recordings with h-Ctrl-lgG, d-Ctrl-lgG, and without lgG (normal solution) showed a normal activity along the entire recording period. The number of synapses/number of animals are as follows: RN, 80/4; h-Ctrl-lgG, 60/3; d-Ctrl-lgG, 60/3; ALS-lgG, 60/3. D, Quantification of normalized MEPP frequency at NMJ in normal solution (RN; open bars) before different treatment (filled bars) with control antibodies ( $\mathrm{h}$-Ctrl-lgG and d-Ctrl-lgG) or antibodies from ALS patients (ALS$\mathrm{lgG}$ ) or other treatments. The sodium channel blocker TTX $(0.5 \mu \mathrm{g} / \mathrm{ml})$ did not prevent ALS-lgG potentiation (TTX+ALS-lgG). Note that labels in which the drug name precedes lgG mean that the drug was present before ALS-lgG-sp had developed, whereas labels in which lgG precedes the drug name indicate that the drug was applied after the ALS-IgG-sp had developed. However, ALS-IgG effect was removed by washing with normal solution after $6.5 \mathrm{~h}$ of incubation (ALS-lgG/Wash 6.5 ) or was prevented by immunoprecipitation of the ALS-lgG with lgG-anti-human (ALS-lgG/Hu) but not with lgGanti-rabbit (ALS-lgG/Rb). Also, synaptic activity was not modified when we used Ctrl-lgG immunoprecipitated with lgG-anti-human (Ctrl-lgG/Hu). Preincubation with aprotinin $(10 \mu \mathrm{g} / \mathrm{ml})$, pepstatin A $(10 \mu \mathrm{M})$, and EGTA $(3 \mathrm{~mm})$ did not prevent ALS-lgG effect (ALS-lgG/Inh). The mean values were calculated from several synapses and several animals and are represented by two numbers (the number of synapses/number of animals). Each open bar shows at least the following: RN, $45 / 3 ; \mathrm{h}-\mathrm{Ctrl}-\mathrm{lgG}$, 45/3; d-Ctrl-lgG, 34/3; ALS-lgG, 55/5; TTX+ALS-IgG, 37/3; ALS-lgG/Inh, 33/3; ALS-lgG/Wash 6.5, 40/4; ALS-lgG/Hu, 32/3; ALS-lgG/Rb, 34/3; and Ctrl-lgG/Hu, 30/3. In graph $D$ and all other graphs, except when stated, bars represent MEPP frequency per minute, and the error bars indicate the SEM normalized to mean frequency before treatment. ${ }^{*} p<0.05$, significantly different from controls by a one-tailed Student's $t$ test.
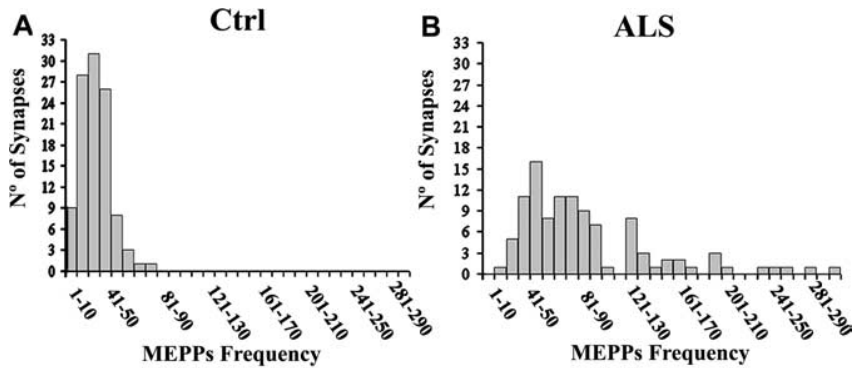

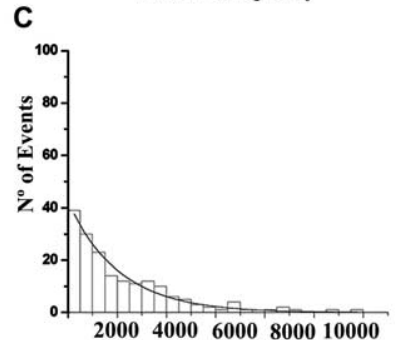

0004000600080001000

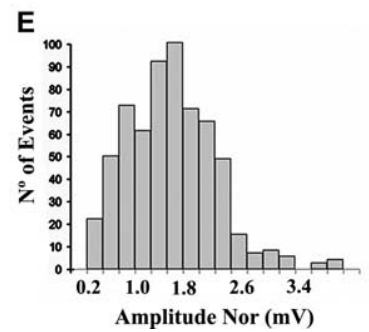

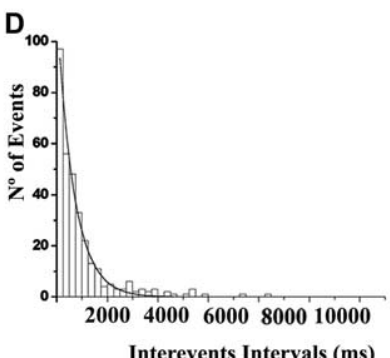

F

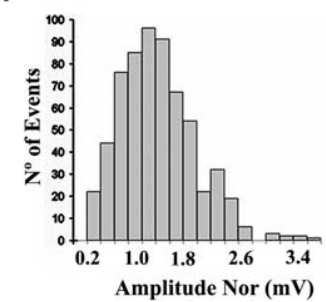

Figure 2. Electrophysiological properties of spontaneous neurotransmission after ALS-IgG application. $\boldsymbol{A}, \boldsymbol{B}$, MEPP frequency distribution, showing an increment in synaptic activity in $\boldsymbol{B}$ (92.75 \pm 5.57$)$ after ALS-IgG application compared with control distribution in $\boldsymbol{A}$ (30.64 \pm 1.32) $(p<0.001) . C, D$, Graphs showing a similar distribution of interevent interval times and fitted to a one-order exponential curve as expected for a stochastic distribution of ACh discharge. $C, r^{2}=0.98, \chi^{2}=2.5 . D, r^{2}=0.98, \chi^{2}=5.23$ ). These properties $(A-D)$ indicated that ALS-IgG increased neurotransmitter release rate but did not modify the release mode. $\boldsymbol{E}, \boldsymbol{F}$, Similar distribution of MEPP amplitude normalized to $-75 \mathrm{mV}(p>0.05)$ after control $(\boldsymbol{E})$ $(1.22 \pm 0.02)$ or ALS $(\boldsymbol{F})(1.24 \pm 0.02)$ antibodies application, which indicated that the quantal size did not change by ALS-IgG. Control graphs were constructed by 450 events from 20 different synapses and those for ALS by 622 events from 10 different synapses.

h-Ctrl-IgG, d-Ctrl-IgG, or without IgG. Mean QC from control and ALS at $20 \mathrm{~Hz}$ was $21.88 \pm 1.61$ and $17.83 \pm 1.53$ and at $40 \mathrm{~Hz}$ was $20.57 \pm 1.73$ and $22.04 \pm 2.05$, respectively $(p>0.05)$.

To determine whether ALS-IgG affects short-term synaptic plasticity, we studied the effect of ALS-IgG on PPF and PTP. ALS-IgG modified neither PPF nor PTP compared with Ctrl-IgG or solution without IgG (data not shown). The values recorded for PPF with an interpulse time of 10,25 , and $50 \mathrm{~ms}$ were as follows: for ALS-IgG, $1.16 \pm 0.04,1.07 \pm 0.03$, and $1.19 \pm 0.06$; and for Ctrl-IgG, $1.03 \pm 0.05,1.09 \pm 0.01$, and $1.07 \pm 0.04(p>$ 0.05). PTP values showed a range from 105.1 to $131.9 \%$ for ALS and from 107.2 to $141.4 \%$ for control antibodies. Thus, these results suggested that ALS-IgG did not affect action potentialdependent synaptic transmission.

\section{ALS-IgG enhances asynchronous and delayed release at 20} and $40 \mathrm{~Hz}$ stimulation

A coordinate fusion of several synaptic vesicles triggers an evoked EPP (synchronous release), and a noncoordinate fusion produces MEPP-like events (asynchronous release). Alterations in synaptic components involved in neurotransmission (i.e., release machinery and $\mathrm{Ca}^{2+}$ homeostasis) could modify the synchronic- 

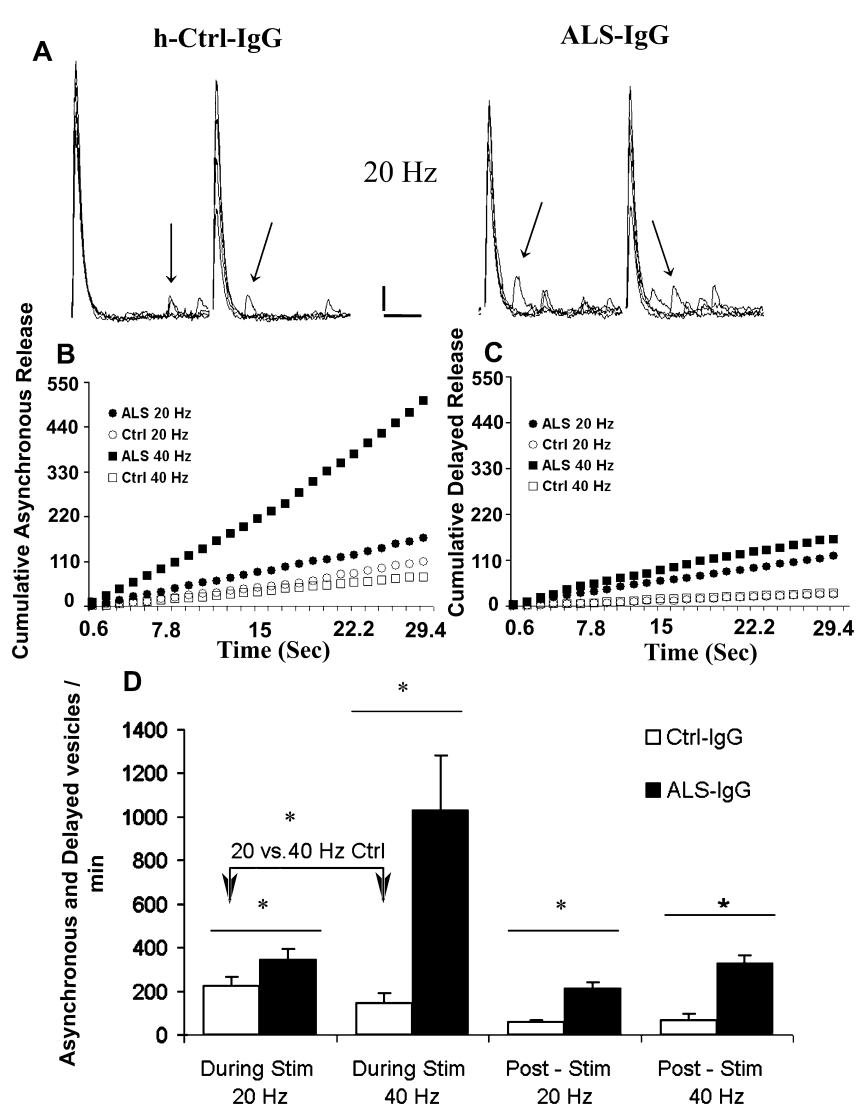

Figure 3. ALS-IgG enhance asynchronous and delayed synaptic vesicle release at 20 and 40 $\mathrm{Hz}$ of stimulation. $\boldsymbol{A}$, Four superimposed sample traces showing EPPs evoked by nerve stimulation at $20 \mathrm{~Hz}$ and asynchronous events between EPPs (arrow) after incubation with Ctrl-lgG or ALS-IgG. The stimulation artifact was blanked for clarity. Calibration: $10 \mathrm{~ms}, 0.5 \mathrm{mV}$. In $\boldsymbol{B}-\boldsymbol{D}$, transmitter release values were quantified every $600 \mathrm{~ms}$ recording. $\boldsymbol{B}$, Cumulative asynchronous neurotransmitter release (during 30 s trains of stimuli at 20 and $40 \mathrm{~Hz}$ ) after ALS-lg (from patient 1) or Ctrl-lgG (from patient A). Each dot represents the mean value obtained from three synapses from three animals. Significant differences between controls and ALS have been observed in both stimulation frequencies (compared by a t test, all of the dots shown) ( $p<0.01$ ). C, Cumulative delayed MEPP-like neurotransmitter release (after $30 \mathrm{~s}$ trains of stimuli at 20 and $40 \mathrm{~Hz}$ ) corresponding to the same experiments shown in $\boldsymbol{B}$. Each dot represents the mean value obtained from three synapses from three animals $(p<0.05)$. D, Bar charts showing quantification of asynchronous (During Stim) and delayed (Post-Stim) events after incubation with Ctrl-IgG (A, B, C, e, d, and f) or with ALS-lgG (1-4). A particular antibody was studied on three synapses from three animals. Each bar represents the mean value and SEM obtained from 12 synapses from 12 animals to ALS antibodies and 18 synapses from 18 animals to control antibodies. Note that asynchronous release was higher at $20 \mathrm{~Hz}$ than at $40 \mathrm{~Hz}$ in $\mathrm{Ctrl}-\mathrm{IgG}$, whereas in ALS-lgG, it was much higher at $40 \mathrm{~Hz}$ than at $20 \mathrm{~Hz}$. ${ }^{*} p<0.05$.

ity of synaptic vesicle fusion increasing asynchronous release (Mochida et al., 1996; David and Barrett, 2003). Because ALS-IgG seems to affect $\mathrm{Ca}^{2+}$ homeostasis, we studied its effect on the asynchronous release that occurs both during and after nerve stimulation (delayed release).

We observed that a $30 \mathrm{~s}$ train of electrical stimulation at 20 and $40 \mathrm{~Hz}$ induced an asynchronous release of $211 \pm 45$ and $146 \pm 49$ MEPPs/min, respectively, in muscles incubated for $4 \mathrm{~h}$ with control antibody (h-Ctrl-IgG and d-Ctrl-IgG) (Fig. $3 A, B$ ). These observations showed a negative correlation between stimulation frequency and asynchronous release $(r=-0.39)(p<0.01)$ (Fig. $3 D)$. Studies at neuromuscular and hippocampal synapses have shown that an increase in the asynchronous release reduces the synchronous release, probably because of the fact that vesicles involved in synchronous and asynchronous release derive from the same source (David and Barrett, 2003; Otsu and Murphy, 2004) (also see Sara et al., 2005).

ALS-IgG-treated muscles stimulated at 20 and $40 \mathrm{~Hz}$ showed a much higher frequency of asynchronous release $(344 \pm 48$ and $1028 \pm 252 \mathrm{MEPPs} / \mathrm{min}$, respectively) and inverted the correlation between the stimulation frequency and asynchronous release $(r=0.87)(p<0.01)$ (Fig. $3 B, D)$. The increment in asynchronous release was time dependent and detectable $\sim 5 \mathrm{~s}$ after the stimulation train started (Fig. $3 B$ ). Interestingly, the increment in asynchronous neurotransmission did not reduce synchronous release at any frequency of stimulation studied (QC at 20 and $40 \mathrm{~Hz}$ ), as could be expected from previous studies (David and Barrett, 2003; Otsu and Murphy, 2004).

ALS-IgG also increased delayed MEPP-like events after a train of action potentials ( $30 \mathrm{~s}$ at 20 or $40 \mathrm{~Hz}$ ). The delayed events in Ctrl-IgG began to return to normal rate after 1-1.5 min, whereas the delayed events in ALS-IgG remained higher (Fig. 3D). Thus, during and after a train of action potentials ( $30 \mathrm{~s}$ at 20 or $40 \mathrm{~Hz}$ ), ALS-IgG promoted asynchronous and delayed release. The ALSIgG effect on asynchronous and spontaneous release, but not on synchronous release, could be because the ALS-IgG affects mechanisms associated with those modes of transmitter release (i.e., intracellular calcium stores) (Narita et al., 2000; David and Barrett, 2003; Otsu and Murphy, 2004) that involve a different type of synaptic vesicles pool (Sara et al., 2005) and distinct release machinery (Hua et al., 1998). Furthermore, at mouse NMJ, a cross talk between synchronous and asynchronous release is apparent only when asynchronous release reaches $\sim 1000$ quanta/s (David and Barrett, 2003), a higher rate than that induced by ALS-IgG ( 1000 quanta/min). Thus, considering the huge size of the synaptic vesicle pool at the MNT and the small number of quanta released asynchronously, ALS-IgG would not be expected to influence the availability of synaptic vesicles for synchronous release in our experiments.

\section{Immunostaining reveals a presynaptic localization of the ALS-IgG molecular target at rodent NMJ}

Our previous experiments strongly suggest that ALS-IgG interacts with a molecular component from the neuromuscular preparation and mediates synaptic potentiation. In fact, it has been suggested that ALS-IgG may be interacting with calcium channels, including non-neuronal VDCCs from skeletal muscle $\left(\mathrm{Ca}_{\mathrm{v} 1.1}, \alpha 1_{\mathrm{S}}\right.$ subunits), thereby altering calcium currents (Delbono et al., 1991; Smith et al., 1992). Furthermore, an interaction has been suggested with the receptor type $\gamma$ for the Fc segment of antibodies (Fc $\gamma \mathrm{R}$ ) (Mohamed et al., 2002). To examine whether ALS-IgG presents immunoreactivity against a molecular target and, if so, where it is located, we studied the binding of ALS-IgG to permeabilized muscle sections and whole nonpermeabilized muscle fibers by immunohistochemistry and confocal microscopy.

ALS-IgG, Ctrl-IgG, or anti-synaptotagmin antibodies were used for indirect immunofluorescence experiments. Double labeling was made by indirect immunostaining (Fig. 4Ad-Af) combined with a direct staining using $\alpha$-bungarotoxin to detect postsynaptic AChRs (Fig. $4 A a-A c$ ) on mouse diaphragm and soleus muscle cross sections.

We studied the immunoreactivity of 13 ALS-IgG and 15 CtrlIgG. We observed that ALS-IgG from 6 of 13 patients (46.15\%) rendered positive labeling, with similar localization at the NMJ. In one case (1 of 13), we observed slight positive staining at NMJ. Furthermore, ALS-IgG from another patient (1 of 13) showed negative staining at NMJ but positive staining in axons. 


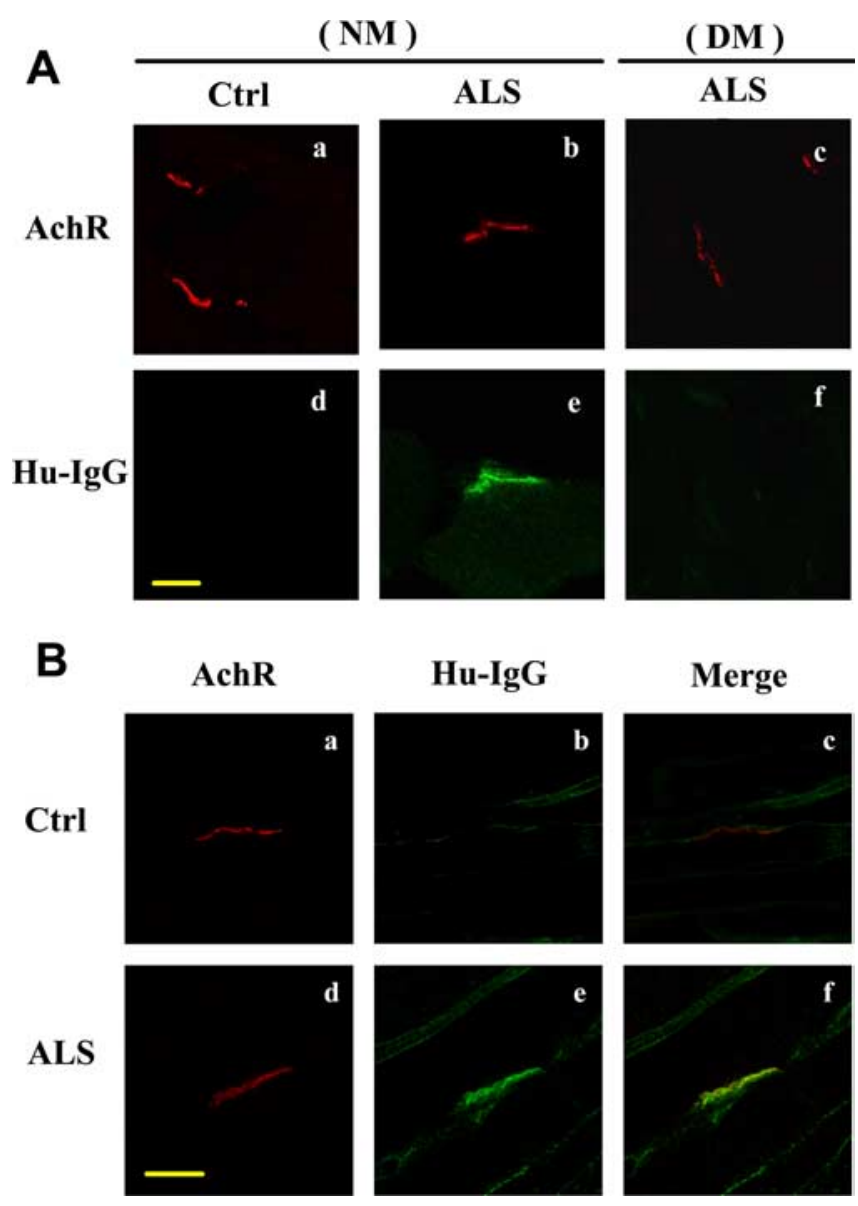

Figure 4. Immunohistochemical detection of the molecular target for ALS-IgG localized at the presynaptic membrane in mouse neuromuscular synapses. Double-fluorescence labeling showing postsynaptic $A C h R$ and immunostaining with human $\operatorname{lgG}(\mathrm{Hu}-\mathrm{lgG}) . A$, Immunostaining detected on soleus muscle cross sections by confocal microscopy. AChRs were detected by $\alpha$-bungarotoxin coupled to tetramethylrhodamine $(\boldsymbol{a}-\boldsymbol{c})$. Anti-human $\lg \mathrm{G}$ coupled to FITC was used in immunostaining with $\mathrm{Hu}$-IgG as primary antibody: $\boldsymbol{d}, \mathrm{Ctrl}-\mathrm{lgG} ; \boldsymbol{e}, \boldsymbol{f}, \mathrm{ALS}-\mathrm{lg}$ G. In left and middle panels $(\boldsymbol{a}, \boldsymbol{b}, \boldsymbol{d}, \boldsymbol{e})$, normal muscles were used (NM), and, in the right panels $(\boldsymbol{c}, \boldsymbol{f})$, denervated muscles were used (DM). Control experiments show AChR localization (a) but negative labeling by $\mathrm{Ctrl}-\operatorname{lgG}(\boldsymbol{d})$. In the middle panels, samples show AChR localization $(\boldsymbol{b})$ and positive labeling by ALS- $\lg G(\boldsymbol{e})$, colocalizing with AChR. ALS-lgG binding in denervated muscles was lost $(\boldsymbol{f})$, whereas $A C h R$ remained at the synapse $(\boldsymbol{c})$, suggesting a presynaptic localization for the molecular target of ALS-IgG. Scale bar, $10 \mu \mathrm{m}$. $\boldsymbol{B}$, Immunostaining detected on whole nonpermeabilized levator auris longus muscles by confocal microscopy. AChRs were detected by $\alpha$-bungarotoxin coupled to tetramethylrhodamine $(\boldsymbol{a}, \boldsymbol{d})$. Anti-human IgG coupled to FITC was used in immunostaining with $\mathrm{Hu}-\mathrm{lgG}$ as primary antibody: $\boldsymbol{b}, \mathrm{Ctrl}-\mathrm{lgG} ; \boldsymbol{e}, \mathrm{ALS}-\mathrm{IgG}$. ALS-IgG staining colocalized with AChR was clearly noticeable, despite that a high background of nonspecific staining was always observed in nonpermeabilized muscle fibers. Scale bar, $20 \mu \mathrm{m}$.

The remaining ALS-IgG ( 5 of 13 ) did not show immunoreactivity at NMJ, axons, or muscle fibers. All Ctrl-IgG (15 of 15) showed negative immunoreactivity against neuromuscular preparations.

Staining revealed a precise labeling at synapses when ALS-IgG was used as primary antibody (Fig. $4 A b, A e$ ) but not when different Ctrl-IgG were used (Fig. $4 A a, A d$ ). Moreover, we observed an exact colocalization of AChRs with the ALS-IgG molecular target and the presynaptic marker synaptotagmin in optic sections of 2 $\mu \mathrm{m}$ (data not shown). It is interesting to note that there is no labeling of muscle fibers, suggesting that, at least on fixed muscles, ALS-IgG does not interact with VDCC $\alpha 1_{\mathrm{S}}$ subunits localized at the muscle fibers. We observed similar results on cross sections of mouse and rat diaphragm and on soleus muscle (data not shown).

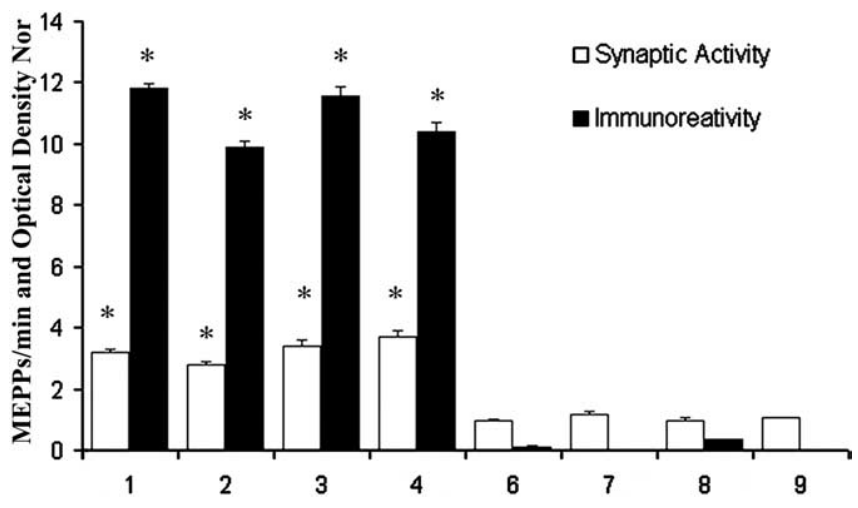

Figure 5. Correlation between ALS-IgG immunoreactivity and ALS-IgG-sp. The graph shows values of MEPP frequency normalized to control as in Figure 1 and values of fluorescence signal after background subtraction and normalization to $100 \mathrm{U}$ of optical density, which represent a minimal fluorescence emission level (close to background level). Values of fluorescence signal were measured as described in Materials and Methods. Numbers on the $x$-axis represent different ALS-IgG with positive (1-4) and negative (6-9) immunoreactivity. Note that only ALS-IgG with positive immunoreactivity also showed an increment in synaptic activity, whereas ALS-IgG with negative immunoreactivity showed normal synaptic activity $(r=0.96)$ and the coefficient of determination $\left(r^{2}=0.92\right)(p<0.01)$. The small open bars for $6-9$ represent normal synaptic transmission.

With the aim of determining whether the molecular target for ALS-IgG was located at nerve terminal or at another NMJ component, such as Schwann cell or muscle fibers, we studied positive ALS-IgG immunoreactivity on cross sections of mouse denervated soleus muscle. Double staining on denervated muscles showed positive labeling at NMJ for AChRs (Fig. 4Ac) but not for ALS-IgG (Fig. $4 A f$ ) or synaptotagmin (data not shown), indicating that the molecular component recognized by ALS-IgG is at MNT (Fig. 4Ac,Af). The negative labeling for synaptotagmin confirmed effective denervation.

To further investigate whether antibody binding site is located on the nerve terminal surface, we performed immunostaining experiments on whole nonpermeabilized muscle fibers (Fig. 4B) using three of the ALS-IgG that had shown positive immunoreactivity in muscle cross sections (Fig. 4A). ALS-IgG showed a positive stain colocalizing with the postsynaptic receptors (Fig. $4 \mathrm{Bd}, \mathrm{Be}$ ), whereas control antibodies did not show specific staining (Fig. $4 \mathrm{Ba}, \mathrm{Bc}$ ). These results strongly indicated that ALS-IgG interacts with a molecule located at the surface of the motor nerve terminals and in turn triggers synaptic modulation.

\section{Correlation between ALS-IgG immunoreactivity and synaptic potentiation mediated by ALS-IgG in neuromuscular synapses}

We have shown that ALS-IgG from $~ 50 \%$ of ALS patients induce synaptic potentiation and positive immunostaining, whereas Ctrl-IgG showed neither synaptic potentiation nor immunoreactivity against MNT. To examine whether synaptic potentiation and positive staining were independent events, we analyzed the correlation between synaptic potentiation and immunostaining in eight different ALS antibodies. None of the ALS-IgG with negative labeling at NMJ modulated synaptic transmission, whereas all ALS-IgG with positive immunoreactivity did. Figure 5 shows mean values of ALS-IgG-sp and immunolabeling of the ALS-IgG studied. Using values of fluorescence signal (independent variable) against mean synaptic activity (dependent variable) from all IgG studied, we estimated the correlation coefficient $(r=0.96)$ and the coefficient of determination $\left(r^{2}=0.92\right)(p<0.01)$. This 
correlation suggested that ALS-IgG-sp is the consequence of ALS-IgG immunoreactivity against MNT. Thus, considering both immunoreactivity and synaptic potentiation studies, we were able to distinguish two different populations of ALS patients. The first contained IgG immunoreactive against MNT and the ability to modulate synaptic transmission, whereas the second did not show any of these features. We will call these two groups "immune-associated ALS" and "idiopathic ALS," respectively. Although there was not a common clinical examination protocol to evaluate the disease progression in each patient, it was possible to determine that there were no clear differences between ALS patients with reacting and nonreacting IgG regarding age at onset ( 53 vs 62 years old), sex (three males/two females vs four males/ two females), patients with bulbar onset (33 vs 25\%), patients with limb onset (67 vs 75\%), time to gastrostomy ( 22 vs 26 months), and time to death or tracheotomy (45 vs 50 months), respectively.

\section{The lack of immunoreactivity by IgG and synaptic potentiation activity in familial ALS}

To evaluate whether immunoreactivity was a consequence of the disease, we examined whether IgG purified from three familial ALS patients (fALS-IgG) showed any immunoreactivity and whether they were capable of modulating spontaneous transmitter release at neuromuscular synapses. We observed that, in diaphragm muscles, fALS-IgG showed no ability to modulate spontaneous synaptic activity, even at an extremely high IgG concentration $(6 \mathrm{mg} / \mathrm{ml})$ and after a prolonged incubation time (7 h). Furthermore, immunostaining revealed the absence of the immunoreactivity of fALS-IgG against NMJ, axons, or muscle fibers in mouse diaphragm and soleus muscles.

\section{Mechanisms underlying ALS-IgG synaptic modulation}

We have shown that ALS-IgG strongly modified the spontaneous frequency of MEPPs in the absence of stimulation, as well as the frequency of asynchronous events during and after stimulation. However, the mechanism underlying this phenomenon needs to be understood. Thus, we concentrated our efforts on studying the signaling pathway responsible for neurotransmitter spontaneous release potentiation induced by four ALS-IgG with positive immunostaining (antibodies from patients 1-4).

\section{Participation of the complement}

Antibodies from Guillain-Barre patients may interact with antigens at MNT and trigger activation of complement (Cp), which produces a high neurotransmitter release rate (Bullens et al., 2002). To evaluate the participation of complement in ALS-IgG$\mathrm{sp}$, we tested whether the addition of Cp boosts ALS-IgG-sp in two experimental conditions: (1) adding $\mathrm{Cp}$ at the time in which ALS-IgG-sp was expected to be apparent, and (2) after ALSIgG-sp was observed. We used normal human sera as a source of $\mathrm{Cp}$ and sera with inactivated complement as a control, as by Bullens et al. (2002). Cp application under conditions 1 and 2 did not boost ALS-IgG-sp. On the contrary, serum with or without $\mathrm{Cp}$ induced an MEPP frequency reduction (data not shown). Furthermore, ALS-IgG treated with the standard procedure for complement inactivation was still capable of inducing synaptic potentiation (RN, $1 \pm 0.09$; ALS-IgG, $2.7 \pm 0.15)(p<0.05)$. We tested four ALS-IgG with positive immunostaining, in which each antibody was studied at least on 30 synapses from three animals. These results did not support the hypothesis that ALSIgG-sp is a complement-mediated phenomenon.
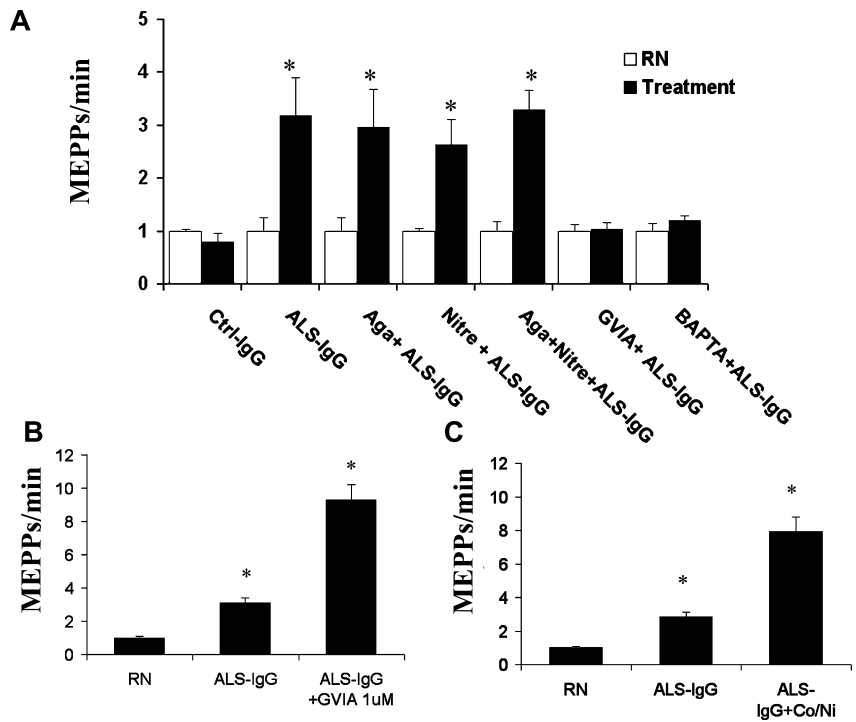

Figure 6. ALS-IgG-sp requires influx of extracellular $\mathrm{Ca}^{2+}$ through N-type VDCCs. $A, \mathrm{P} / \mathrm{Q}$ - or L-type VDCC blockers did not prevent ALS-IgG-induced synaptic potentiation when used individually (Aga + ALS-IgG and Nitre+ALS-IgG, respectively) or simultaneously $($ Aga + Nitre + lgG), whereas N-type VDCC blocker prevented ALS-lgG effect (GVIA + ALS-lgG). The labels should be interpreted as stated in Figure 1. $\omega$-Agatoxin IVA (Aga; $100 \mathrm{nm),} \mathrm{nitren-}$ dipine (Nitre; $10 \mu \mathrm{M}$ ), and $\omega$-conotoxin GVIA (GVIA; $1 \mu \mathrm{m}$ ) were used to block P/Q-, L-, and $\mathrm{N}$-type VDCCs, respectively. Cell-permeable BAPTA-AM $(10 \mu \mathrm{m})$ prevented ALS-IgG-sp (BAPTA+ALS-IgG). Quantification of normalized MEPP frequency at NMJ in normal solution (RN; open bars) before different treatment (filled bars) with control antibodies ( $\mathrm{h}-\mathrm{Ctrl}-\mathrm{IgG}$ and $\mathrm{d}$-Ctrl-IgG) or antibodies from ALS patients (ALS-IgG) or other treatments. B, Bar chart representing synaptic activity measured consecutively in normal solution (RN), after $100 \mu \mathrm{g} / \mathrm{ml}$ ALS-IgG application (ALS-IgG), and after $\omega$-conotoxin GVIA addition (ALS-IgG + GVIA). To evaluate a continuous requirement of $\mathrm{Ca}^{2+}$ influx through $\mathrm{N}$-type VDCCs, the effect of $\omega$-conotoxin GVIA $1 \mu \mathrm{M}$ was tested by toxin application when ALS-IgG-sp was evident. C, Bar chart representing synaptic activity measured consecutively in normal solution (RN), after $100 \mu \mathrm{g} / \mathrm{ml}$ ALS-lgG application (ALS-lgG), and after addition of a mix of $\mathrm{C}^{2+}$ and $\mathrm{Ni}^{2+}(300 \mu \mathrm{m}$ each) (ALS-lgG $+\mathrm{Co} / \mathrm{Ni}$ ), to evaluate a continuous requirement of $\mathrm{Ca}^{2+}$ influx through $\mathrm{N}$-type VDCC or any other VDCC. Note that, in $\boldsymbol{B}$ and $\boldsymbol{C}$, calcium channel blockers were applied when ALSIgG-sp was evident. Addition of neither GVIA nor $\mathrm{Co}^{2+}$ and Ni ${ }^{2}$ interrupt ALS-lgG-sp, indicating absence of continuous $\mathrm{Ca}^{2+}$ requirement through $\mathrm{N}$-type VDCC. The number of synapses studied is expressed as in Figure 1. The number of synapses per number of animals is as follows (open bars). $\boldsymbol{A}, \mathrm{RN}, 45 / 3$; ALS-IgG, 66/6; Nitre + ALS-IgG, 50/4; Aga + ALS-IgG, 36/3; and GVIA + ALS$\operatorname{lgG}, 65 / 5 . \boldsymbol{B}, \mathrm{RN}, 33 / 3 ; \mathrm{ALS}-\operatorname{lgG}, 34 / 3$; and ALS-IgG + GVIA, 35/3. C, RN, 34/3; ALS-IgG, 32/3; and $\mathrm{ALS}-\lg \mathrm{G}+\mathrm{Co} / \mathrm{Ni}, 45 / 3$.

\section{ALS-IgG-induced synaptic potentiation requires an initial $\mathrm{Ca}^{2+}$ influx through a $\mathrm{Ca}_{\mathrm{v}} 2.2$ calcium channel}

At adult neuromuscular junction, P/Q VDCC mediates transmitter release (Katz et al., 1997). However, other types of VDCCs are also present at NMJ (Pagani et al., 2004). To further investigate the mechanisms underlying ALS-IgG-sp, we examined whether the ALS-IgG effect requires $\mathrm{Ca}^{2+}$ influx by using specific blockers to P/Q-, L-, and N-type VDCCs.

L-type or P/Q-type VDCC blockers (10 mm nitrendipine or $100 \mathrm{~nm} \omega$-agatoxin IVA, respectively) did not prevent ALS-IgG effect when they were used either alone or jointly (Fig. 6A). In contrast, the N-type VDCC blocker GVIA ( $1 \mu \mathrm{M})$ completely prevented the ALS-IgG effect (Fig. 6A). Additionally, the permeable $\mathrm{Ca}^{2+}$ chelator BAPTA-AM $(10 \mu \mathrm{M})$ prevented ALS-IgG-sp (Fig. $6 A$ ). To evaluate whether the ALS-IgG-sp requires a continuous $\mathrm{Ca}^{2+}$ influx through N-type VDCCs $\left(\mathrm{Ca}_{\mathrm{v} 2.2}\right)$, we blocked $\mathrm{Ca}_{\mathrm{v}} 2.2$ channels after the ALS-IgG effect was established. Surprisingly, the $\mathrm{Ca}_{\mathrm{v}} 2.2$ VDCC blocker GVIA $(1 \mu \mathrm{M})$ was not able to reverse the ALS-IgG effect (Fig. 6B, Table 1). Although GVIA blocks $\mathrm{Ca}_{\mathrm{v}} 2.2$ channels by pore occlusion and does so almost 
Table 1. Common mechanisms participate in synaptic potentiation mediated by IgG from different ALS patients

\begin{tabular}{|c|c|c|c|c|c|c|c|c|c|c|}
\hline \multirow[b]{2}{*}{ ALS-IgG } & \multirow[b]{2}{*}{ RN } & \multirow[b]{2}{*}{$\lg G$} & \multirow{2}{*}{$\begin{array}{l}\text { GVIA + } \\
\lg G\end{array}$} & \multirow{2}{*}{$\begin{array}{l}\text { U73 + } \\
\lg G\end{array}$} & \multirow{2}{*}{$\begin{array}{l}\text { Ryan + } \\
\lg G\end{array}$} & \multirow{2}{*}{$\begin{array}{l}A P B+ \\
\lg G\end{array}$} & \multicolumn{4}{|c|}{ After ALS-IgG-sp and application of } \\
\hline & & & & & & & + GVIA & + Ryan & $+\mathrm{APB}$ & + Ryan/APB \\
\hline 1 & $1 / 0.16$ & $3.11 / 0.39^{a}$ & $0.88 / 0.21$ & $1.05 / 0.11$ & $0.83 / 0.16$ & $1.17 / 0.28$ & $3.02 / 0.22^{a}$ & $2.18 / 0.02^{a}$ & $3.06 / 0.04^{a}$ & $1.28 / 0.11$ \\
\hline 2 & $1 / 0.09$ & $2.34 / 0.32^{a}$ & $0.77 / 0.14$ & $1.17 / 0.09$ & $1.11 / 0.14$ & $1.20 / 0.04$ & $2.86 / 0.18^{a}$ & $2.38 / 0.04^{a}$ & $3.86 / 0.25^{a}$ & $1.27 / 0.06$ \\
\hline 3 & $1 / 0.07$ & $2.79 / 0.43^{a}$ & $1.07 / 0.07$ & $0.84 / 0.08$ & $0.97 / 0.03$ & $1.04 / 0.09$ & $5.34 / 1.81^{a}$ & $2.24 / 0.13^{a}$ & $2.11 / 0.08^{a}$ & $1.16 / 0.04$ \\
\hline 4 & $1 / 0.08$ & $3.55 / 0.98^{a}$ & $1.15 / 0.06$ & $1.06 / 0.05$ & $0.95 / 0.07$ & $1.13 / 0.06$ & $3.31 / 0.46^{a}$ & $2.37 / 0.13^{a}$ & $2.34 / 0.09^{a}$ & $1.16 / 0.02$ \\
\hline
\end{tabular}

Synaptic activity recorded in normal solution (RN), with ALS-lgG (IgG), and with ALS-lgG before and after application of different inhibitors (i.e., GVIA + IgG). Application of inhibitors before ALS-IgG is represented as follows: GVIA + IgG, $\mathrm{U} 73+\mathrm{lgG}$, Ryan + lgG, and APB + IgG. Application of inhibitors after ALS-lgG is represented as follows: + GVIA, + Ryan, + APB, and + Ryan/APB. Addition of the inhibitor after ALS-IgG-sp means that the inhibitor was added after ALS-IgG-sp had developed. Values represent mean \pm SEM MEPP frequency per minute normalized to control. Each value was obtained by studying at least 30 synapses from three different animals. Numbers $1-4$ indicate four different ALS-IgG with positive immunolabeling. Statistical analysis was made by a one-tail Student's $t$ test. U73, U73122; Ryan, ryanodine.

${ }^{a}$ Mean value significantly different from control, $p<0.05$.

irreversibly (Doughty et al., 1998), these results could be explained by a competitive interaction between the toxin and ALSIgG. Thus, we examined this hypothesis by using GVIA at high concentration $(3 \mu \mathrm{M})$, but GVIA neither interrupted ALS-IgG-sp nor prevented the increment in synaptic activity dependent on incubation time (Fig. 1C). A second possibility examined was that ALS-IgG might induce a conformational change over $\mathrm{Ca}_{\mathrm{v}} 2.2$ channels, blocking the interaction between the toxin and the $\mathrm{Ca}_{\mathrm{v}} 2.2$ channels. Likewise, the interaction between the toxin and the channel would not allow that of the ALS-IgG with its molecular target, and both would be mutually excluded. To examine this hypothesis, we proceeded to treat three mouse diaphragm muscles with GVIA or vehicle and, thereafter, fixed and processed muscles for immunohistochemistry. Pretreatment with GVIA did not preclude immunostaining when ALS-IgG were used as primary antibody, given the same labeling on the treated and nontreated muscles (data not shown). In addition, we tested that a mix of $\mathrm{Co}^{2+}(300$ $\mu \mathrm{M})$ and $\mathrm{Ni}^{2+}(300 \mu \mathrm{M})$ is effective to block VDCCs and reverse MEPP frequency increment induced by $10 \mathrm{mM} \mathrm{K}{ }^{+}$. However, $\mathrm{Co}^{2+} / \mathrm{Ni}^{2+}$ application was ineffective to reverse the ALS-IgG effect (Fig. 6C). These experiments with the $\mathrm{Ca}_{\mathrm{v}} 2.2$ channel blocker denote the absence of competitive and noncompetitive interaction between GVIA and ALS-IgG. Jointly, these molecular and physiological studies revealed that ALS-IgG did not interact with $\mathrm{Ca}_{\mathrm{v}} 2.2$ channels and that ALS-IgG-sp requires an initial influx of $\mathrm{Ca}^{2+}$ through $\mathrm{Ca}_{\mathrm{v}} 2.2$, channels but not a constitutive $\mathrm{Ca}^{2+}$ influx. Additionally, these findings showed that the $\mathrm{Ca}^{2+}$ signal from $\mathrm{Ca}_{\mathrm{v}} 2.2$ channels might somehow regulate neurotransmitter release at mouse neuromuscular synapses (Protti et al., 1991).

The ALS-IgG effect requires activation of phospholipase C and involves continuous and simultaneous participation of 1,4,5-trisphosphate and ryanodine-sensitive receptors

Our previous findings suggested that the ALS-IgG effect requires not only $\mathrm{Ca}^{2+}$ influx through $\mathrm{Ca}_{\mathrm{v}} 2.2$ channels but also a concomitant activation of signaling pathways when the ALS-IgG-sp became independent of $\mathrm{Ca}^{2+}$ influx. Thus, activation of some pathways may explain the high spontaneous transmitter release independent of $\mathrm{Ca}^{2+}$ influx through $\mathrm{Ca}_{\mathrm{v}} 2.2$ (Fig. 6). To examine
B

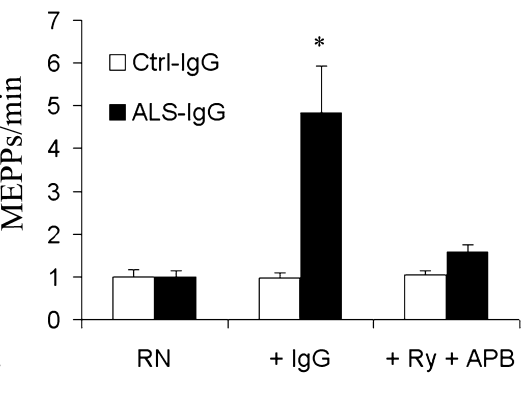

Figure 7. ALS-IgG-mediated synaptic potentiation was dependent on $P L C, I P{ }_{3} R$, and RyR signaling pathways. A, Quantification H7+ALS-lgG), PI3-K using wortmannin (1 $\mu \mathrm{M})$ (Wort+ALS-lgG), or protein synthesis using cycloheximide (by $10 \mu \mathrm{g} / \mathrm{ml}$ ) (Cyclos ineffective in preventing ALS-lgG potentiation. However, inhibition of PLC (by $10 \mu \mathrm{m} U 73122)(\mathrm{U} 73+\mathrm{ALS}$ types of receptors are necessary for ALS-IgG-sp. The number of synapses tested is expressed as in Figure 1 . For $\boldsymbol{A}$, each open bar shows at least the following: RN, 45/3; ALS-IgG, 30/3; H7+ALS-IgG, 30/2; Wort + ALS-lgG, 28/2;U73 + ALS-lgG, 45/3; Ry + ALS$\lg G, 24 / 2 ; A P B+A L S-\lg G, 36 / 3$. For $\boldsymbol{B}, \mathrm{RN}, 30 / 3 ;$ ALS-IgG, 30/3; ALS-lgG+Ry/APB, 36/3.

this hypothesis, we tested several pathways that may be involved in the ALS-IgG potentiation phenomenon. The best characterized signaling pathways, which may lead to synaptic potentiation, are those that involve intracellular $\mathrm{Ca}^{2+}$ mobilization and also pathways that involve VDCC activation. Intracellular $\mathrm{Ca}^{2+}$ mobilization could take place through 1,4,5-trisphosphate receptors ( $\left.\mathrm{IP}_{3} \mathrm{Rs}\right)$ and ryanodine receptors (RyRs), and VDCC activation could take place through protein kinase $\mathrm{C}$ ( $\mathrm{PKC}$ ) or cAMP-dependent protein kinase (PKA) (Berridge et al., 2003). Because $\mathrm{IP}_{3} \mathrm{R}$ and $\mathrm{PKC}$ may be under the control of phospholipase C (PLC), we began studying whether the inhibition of PLC by U73122 (1-[6[[(17 $\beta)$-3-methoxyestra1,3,5(10)-trien-17-yl] amino] hexyl]-1 H-pyrrole-2,5-dione) (10 $\mu \mathrm{M})$, a specific PLC inhibitor, affected ALS-IgG-sp. Inhibition of PLC prevented but did not interrupt the ALS-IgG effect (Fig. 7A). We also observed that orthovanadate $(20 \mu \mathrm{M})$, a specific tyrosine phosphatases inhibitor, may induce an ALS-IgG-sp-like effect with similar magnitude and time course. The ability of the orthovanadate to mimic ALS-IgG-sp is consistent with the recruitment of tyrosine phosphorylation necessary for PLC activation in ALS-IgG-sp.

Activated PLC can hydrolyze phosphatidylinositol 4,5bisphosphate, generating the second messengers diacylglycerol (DAG) and $\mathrm{IP}_{3}$. Both DAG and $\mathrm{IP}_{3}$ in the presence of $\mathrm{Ca}^{2+}$ can activate $\mathrm{PKC}$ and $\mathrm{IP}_{3} \mathrm{R}$, respectively, leading to $\mathrm{a}\left[\mathrm{Ca}^{2+}\right]_{\mathrm{i}}$ incre- 
ment (Berridge et al., 2003). To define the PKC or $\mathrm{IP}_{3} \mathrm{R}$ contribution, we evaluated the effect of inhibiting these pathways. Inhibition of PKC using H7 [1-(5-isoquinolinesulfonyl)-2methylpiperazine dihydrochloride] $(100 \mu \mathrm{M})$, a specific serine/ threonine inhibitor, did not prevent the ALS-IgG-sp effect (Fig. $7 A$ ), whereas $A P B$, an $\mathrm{IP}_{3} \mathrm{R}$ antagonist, did (Fig. $7 A$ ). However, $\mathrm{IP}_{3} \mathrm{R}$ antagonist did not reverse the ALS-IgG effect when the potentiation has already developed.

To examine the involvement of RyR (O'Shaughnessy et al., 1998 ) in ALS-IgG-sp, we used ryanodine at a high concentration $(20 \mu \mathrm{M})$ as antagonist for RyR. We observed absolute prevention of ALS-IgG-sp (Fig. 7A). Again, once ALS-IgG-sp had developed, ryanodine application did not reverse synaptic activity to basal level (Fig. 7A). However, the magnitude of ALS-IgG-sp after ryanodine application $(2.29 \pm 0.08 \mathrm{MEPP} / \mathrm{min})$ compared with ALS-IgG-sp without ryanodine $(3.95 \pm 0.42 \mathrm{MEPPs} / \mathrm{min})$ suggested that ryanodine prevented full synaptic potentiation. Nevertheless, using both RyR and $\mathrm{IP}_{3} \mathrm{R}$ antagonists together, we observed a reversal of ALS-IgG-sp. Regardless of the ALS-IgG used (from any patient), reversal of synaptic activity was complete in $41.7 \%$ of the experiments ( 5 of 12 ), showing a $92.5 \%$ mean reversal. The magnitude of the reversal showed a negative correlation with the magnitude of ALS-IgG-sp reached (Fig. 7B, Table 1). ALS-IgG-induced $\mathrm{Ca}^{2+}$ release from intracellular $\mathrm{Ca}^{2+}$ stores would be consistent with an increase of spontaneous and asynchronous release but not an alteration in synchronous release because (1) enhancement of spontaneous and asynchronous release is feasible by $\mathrm{Ca}^{2+}$ release through RyR (Narita et al., 2000; Angleson and Betz, 2001), and (2) in association with an increased asynchronous release, QC is only depressed when asynchronous release is massive $(\sim 1000$ quanta/s) (David and Barrett, 2003) or when the stimulation at high frequency is longer than 2 min (Narita et al., 2000).

Prevention of ALS-IgG effect by inhibition of RyR or $\mathrm{IP}_{3} \mathrm{R}$ indicated that these receptors were necessary to activate an increment in transmitter release in both MEPP frequency (Fig. 7A) and asynchronous release (data not shown). Moreover, the requirement of inhibition of both receptors to reverse the synaptic potentiation indicated that both $\mathrm{RyR}$ and $\mathrm{IP}_{3} \mathrm{R}$ contributed to its maintenance.

Together with activation of PLC, the phosphoinositide 3-kinase (PI3-K) pathway may also be activated (Robidoux et al., 1998) and may regulate synaptic potentiation (Yang et al., 2001) and neurotransmitter release by production of inositol 3,4,5-trisphosphate (Schiavo et al., 1996). We examined the participation of PI3-K in ALS-IgG potentiation by using specific PI3-K inhibitors. Application of neither wortmannin $(1 \mu \mathrm{M})$ (Fig. 7A) nor LY294002 [2-(4morpholinyl)-8-phenyl-1(4H)-benzopyran-4-one] (5 $\mu \mathrm{M})$ had any effect on ALS-IgG-sp. In fact, LY294002 increased the synaptic activity by itself (data not shown), which is consistent with previous observations (Rizzoli and Betz, 2002). Furthermore, because ALSIgG-sp required a long incubation time, we examined whether protein synthesis was required during the incubation. The protein synthesis inhibitor cycloheximide $(10 \mu \mathrm{g} / \mathrm{ml})$ did not alter ALS-IgG-sp (Fig. 7A).

Our pharmacological results suggested that ALS-IgG-sp was dependent on $\left[\mathrm{Ca}^{2+}\right]_{\mathrm{i}}$, which can be inferred from MEPPs by using an appropriate Hill coefficient and half-saturation value $\left(K_{\mathrm{d}}\right)$ (Angleson and Betz, 2001). Thus, using previous data of $\left[\mathrm{Ca}^{2+}\right]_{\mathrm{i}}$ dependence of MEPP frequency (Angleson and Betz, 2001), we estimated the time course of ALS-IgG-induced $\mathrm{Ca}^{2+}$ increment in MNT. This approach showed that, after 4 and $8 \mathrm{~h}$ of incubation, ALS-IgG increased $\left[\mathrm{Ca}^{2+}\right]_{\mathrm{i}}$ to $\sim 200 \mathrm{nM}$ and $8 \mu \mathrm{M}$, respectively.

Additionally, these results provide physiological evidence for a functional coupling between N-type VDCC, PLC, $\mathrm{IP}_{3} \mathrm{R}$, and RyR in neuromuscular synapses. We confirmed these results by using antibodies purified from four ALS patients with positive immunostaining. Results from the most relevant experiments are summarized in Table 1.

\section{Discussion}

\section{Significance of autoantibodies in ALS}

We have shown that IgG from a group of ALS patients binds to MNT, resulting in potentiation of spontaneous and asynchronous release. Several pieces of evidence supported this conclusion. First, protease inhibitors did not preclude ALS-IgG-sp (Fig. $1 D)$ (Nyormoi, 1996). Second, immunoprecipitation using antihuman, but not anti-rabbit, antibodies prevented ALS-IgG-sp (Fig. 1D). Third, these results were consistent with the purity of the purified IgG tested by SDS-PAGE. Fourth, ALS-IgG effect is unlikely to be the result of nerve terminal degeneration because it was reversed after as long as $6.5 \mathrm{~h}$ exposure (Figs. $1 D, 7 B$ ). Fifth, ALS-IgG interacts with an antigen present in the external surface of the MNT (Fig. 4). Sixth, a high correlation supported that ALS-IgG-sp was a consequence of immunoreactivity (Fig. 5).

The lack of effect of pepstatin A (a protease inhibitor and also a potent inhibitor of $\mathrm{Fc} \gamma \mathrm{R}$ ) (Athanassakis et al., 2000) on ALSIgG-sp is in disagreement with the proposed interaction between ALS-IgG and Fc $\gamma$ R (Mohamed et al., 2002). Furthermore, if ALSIgG-sp were the consequence of an interaction between ALS-IgG and $\mathrm{F}_{\mathrm{c}} \gamma \mathrm{R}$, we should have observed positive labeling with all of the IgG tested, including Ctrl-IgG (Ober et al., 2001). Moreover, no reactivity was observed with any of the IgG used at vascular endothelium, in which Fc $\gamma \mathrm{R}$ is highly enriched (Medesan et al., 1998). Immunostaining observed with ALS-IgG therefore indicates that the Fab segment mediated the antigen-antibody interaction.

Presence of autoantibodies in sera of ALS patients has been thought to be a consequence of the disease (Drachman, 2000). Here, we presented evidence to the contrary. The hypothesis predicts that the antibodies generated in ALS patients would be immunoreactive against a wide variety of molecules. We, however, detected immunoreactivity against specific neuronal components with specific localization (Fig. 4). Also, although patients with fALS showed virtually the same clinical and degenerative features as sporadic ALS patients, the IgG purified from fALS patients, as well as $\sim 50 \%$ of the ALS-IgG, showed no immunoreactivity and did not induce synaptic potentiation (Fig. $1 \mathrm{~A}$ ).

Hence, consistent with the notion that ALS is a syndrome, our results revealed that, in $\sim 50 \%$ of ALS patients, MNTs constitute a target for autoimmune response (Vincent, 1999), whereas in the remaining ALS patients, different mechanisms may be involved (Figs. $1 A, 5$ ). Thus, staining assays can be instrumental to distinguishing two groups of ALS patients, which must be considered differently in future studies and therapeutic approaches.

\section{Signaling pathways regulating ALS-IgG-induced synaptic potentiation}

Controversial evidence has been presented about an interaction between ALS-IgG and VDCCs (Ordonez and Sotelo, 1989; Kimura et al., 1994; R. G. Smith et al., 1994; Arsac et al., 1996) and their capability to modify $\mathrm{Ca}^{2+}$ currents. However, the effect of ALS-IgG on $\mathrm{Ca}^{2+}$ currents seems to be dependent on the cellular type studied. For example, a decrease of $\mathrm{Ca}^{2+}$ currents has been 
reported in muscle fibers, granule cells, and dorsal root ganglion cells (Delbono et al., 1991; Zhainazarov et al., 1994; Yan et al., 1997), as well as an increase of currents in cell line VSC4.1 and Purkinje cells (Llinas et al., 1993; Mosier et al., 1995). Although patients with ALS may generate diverse antibodies against different types of VDCCs, those works did not support this hypothesis. Although we did not find any satisfactory explanation for these controversial facts, it is possible to speculate that, in some cases, ALS-IgG may be interacting with a receptor physically and functionally associated with VDCCs, as observed for $\mathrm{Ca}_{\mathrm{v}} 2.2$ and G-protein-coupled receptor (McEnery et al., 1994).

Our studies of signaling pathways showed that ALS-IgG-sp requires $\mathrm{Ca}^{2+}$ influx through $\mathrm{Ca}_{\mathrm{v}} 2.2$ channels but not a constitutive $\mathrm{Ca}^{2+}$ influx or $\mathrm{Ca}^{2+}$ influx through other types of VDCCs (Fig. 6A). Experiments of competitive and noncompetitive interaction between ALS-IgG and GVIA revealed no interaction between them and showed that, after ALS-IgG-sp started, it became independent of $\mathrm{Ca}^{2+}$ influx (Fig. 6). This conclusion was confirmed by $\mathrm{Co}^{2+} / \mathrm{Ni}^{2+}$ application during ALS-IgG-sp, which blocks all VDCCs (Fig. 6). Strikingly, these results suggested that ALS-IgG did not interact with $\mathrm{Ca}_{\mathrm{v}} 2.2$ channels, in agreement with previous immunoprecipitation and ELISA results (Arsac et al., 1996). Furthermore, $\mathrm{Ca}_{\mathrm{v}} 2.2$ channels remain in denervated muscles (Day et al., 1997; Pagani et al., 2004), whereas ALS-IgG molecular target disappears completely after denervation (Fig. 4).

Additionally, ALS-IgG consistently increased transmitter release in different NMJ preparations, including diaphragm muscle (Uchitel et al., 1988; O'Shaughnessy et al., 1998; Mohamed et al., 2002; Muchnik et al., 2002) and levator auris muscle of the mouse (Uchitel et al., 1992; Fratantoni et al., 2000) and guinea pig diaphragm muscle (O. D. Uchitel, unpublished results) and even in hippocampal cellular culture (Andjus et al., 1997). It did not increase transmitter release, however, in PC12 cells (Offen et al., 1998) and mouse extraocular NMJ (Mosier et al., 2000). These results indicate that the ALS-IgG effect is not restricted to the NMJ and is not specific for a particular type of skeletal muscle NMJ. This hypothesis is supported by the specific alteration of motoneurons $\left(\left[\mathrm{Ca}^{2+}\right]_{\mathrm{i}}\right.$ increment) after systemic injection of ALS-IgG (Engelhardt et al., 1995; Pullen and Humphreys, 2000; Pullen et al., 2004) but no $\left[\mathrm{Ca}^{2+}\right]_{i}$ increment in synaptosomes from cortex (Thomas and Dunn, 1997). This indicates that ALSIgG somehow exhibits specificity to affect motoneurons. Accordingly, immunoreactivity against MNT (Fig. 4) may explain such cellular specificity.

We identified that PLC, RyR, and $\mathrm{IP}_{3} \mathrm{R}$ were fundamental in ALS-IgG-sp activation and that RyR and $\mathrm{IP}_{3} \mathrm{R}$ were active simultaneously to maintain ALS-IgG-sp (Fig. 7) (supplemental Fig. S1, available at www.jneurosci.org as supplemental material). This pathway may account for $\left[\mathrm{Ca}^{2+}\right]_{\mathrm{i}}$ increment in motoneurons after a systemic injection of ALS-IgG (Engelhardt et al., 1995; Pullen and Humphreys, 2000; Pullen et al., 2004).

These results led us to question the functional role of the $\mathrm{Ca}^{2+}$ signals through $\mathrm{Ca}_{\mathrm{v}} 2.2$ channels suggested by the blocking effect of GVIA. A Ca ${ }^{2+}$ signal through $\mathrm{Ca}_{\mathrm{v}} 2.2$ channels close to endoplasmic reticulum (ER) may cooperate in the opening of individual $\mathrm{IP}_{3} \mathrm{R}$ and RyR. $\mathrm{Ca}^{2+}$ through $\mathrm{Ca}_{\mathrm{v}} 2.2$ channels may regulate positively $\mathrm{Ca}^{2+}$ store or may even stimulate a higher $\left[\mathrm{Ca}^{2+}\right] \mathrm{ER}$ luminal level, which results in sensitization of RyR and $\mathrm{IP}_{3} \mathrm{R}$ (Berridge et al., 2003). This hypothesis is in agreement with our observation that the $\mathrm{Ca}^{2+}$ chelator BAPTA-AM prevented ALSIgG-sp and with previous reports (Stoop and Poo, 1995) (for hypothetical model, see supplemental Fig. S1, available at www. jneurosci.org as supplemental material).
Potential functional relevance of ALS-IgG-sp in ALS patients We have shown that, while we maintained the ALS-IgG stimulus, ALS-IgG-sp persisted and became more enhanced as time proceeded (Fig. 1C). Our observations provide evidence that ALSIgG may trigger and maintain a $\left[\mathrm{Ca}^{2+}\right]_{\mathrm{i}}$ increment. Thus, a continuous $\mathrm{Ca}^{2+}$ release may constitute a signal consistent with a disturbance of $\mathrm{Ca}^{2+}$ storage and signaling in motoneurons.

There is sound evidence that $\mathrm{Ca}^{2+}$ store alteration can lead to ER stress with consequent protein misfolding, as has been reported in ALS patients (Strong, 2003). ER stress caused by alterations in $\mathrm{Ca}^{2+}$ transport system $\left(\mathrm{Ca}^{2+}\right.$-ATPase and $\left.\mathrm{IP}_{3} \mathrm{R}\right)$ can be linked directly to caspase activation (Jayaraman and Marks, 1997; Orrenius et al., 2003). In addition, a persistent $\mathrm{Ca}^{2+}$ release from RyR and $\mathrm{IP}_{3} \mathrm{R}$ leads to cytosolic $\mathrm{Ca}^{2+}$ overload. However, when $\left[\mathrm{Ca}^{2+}\right]_{\mathrm{i}}$ rises, mitochondrial $\mathrm{Ca}^{2+}$ uptake overcomes this situation, because mitochondria, ER, and VDCCs can form a functional unit attributable to their proximity (Rizzuto et al., 1993). Interestingly, ALS-IgG enhanced asynchronous release, which is reduced by mitochondrial $\mathrm{Ca}^{2+}$ uptake (David and Barrett, 2003). Thus, the ALS-IgG-sp is likely to affect both ER and mitochondrial homeostatic $\mathrm{Ca}^{2+}$ control (Engelhardt et al., 1995), which may lead to caspase activation (i.e., caspase-3 activation through ER, caspase-12 or mitochondrial, caspase-9) (Orrenius et al., 2003; Demestre et al., 2005). In agreement with these assumptions, apoptosis mediated by mitochondrial dysfunction has been reported in ALS patients (Strong, 2003).

After a transient insult at NMJ, denervation, sprouts, and reinnervation can be observed (Maselli et al., 1993; Cui et al., 2004). However, with such a persistent insult as ALS-IgG in an ALS patient, emerging MNT during reinnervation could be target for ALS-IgG and become dysfunctional once more, establishing a catastrophic cellular economy (Orrenius et al., 2003). Accordingly, in both ALS patients as in animals treated with ALS-IgG, we can expect MNT dysfunction and then structural alterations in motoneurons (Uchitel et al., 1992; Pullen et al., 2004).

Interestingly, ALS-IgG did not affect QC, contrasting with QC evaluated at low-stimulation frequency (O'Shaughnessy et al., 1998). However, ALS-IgG enhanced asynchronous release at physiological stimulation frequencies. Unlike control recording, asynchronous release was much higher at $40 \mathrm{~Hz}$ than at $20 \mathrm{~Hz}$. Strikingly, these observations predicted that a higher rate of action potential-evoked neurotransmission might promote a higher degree of intracellular $\mathrm{Ca}^{2+}$ deregulation in motoneurons of ALS patients. Thus, because strength in muscle contraction is achieved by an increment in firing frequency and recruitment of motor units (Norris and Gasteiger, 1955), motoneurons involved in heavier tasks could be expected to be preferentially affected in ALS and to be reflected in the onset and/or progression pattern of ALS. Accordingly, it is interesting to note that limb onset is found in $65-80 \%$ of ALS cases (Brooks et al., 1995). Additionally, in younger ALS patients, there is a preferential right arm onset (Brooks et al., 1995), as could be expected if motoneurons involved in heavier tasks were preferentially affected in ALS. Also, intensive physical activity has been associated with ALS (Chio et al., 2005). Finally, these results stress the relevance of identifying specific mechanisms for immune-associated and idiopathic ALS, together with detailed clinical studies to obtain more accurate interpretations of clinical trials.

\section{References}

Andjus PR, Stevic-Marinkovic Z, Cherubini E (1997) Immunoglobulins from motoneurone disease patients enhance glutamate release from rat hippocampal neurones in culture. J Physiol (Lond) 504:103-112. 
Angleson JK, Betz WJ (2001) Intraterminal $\mathrm{Ca}^{2+}$ and spontaneous transmitter release at the frog neuromuscular junction. J Neurophysiol 85:287-294.

Arsac C, Raymond C, Martin-Moutot N, Dargent B, Couraud F, Pouget J, Seagar M (1996) Immunoassays fail to detect antibodies against neuronal calcium channels in amyotrophic lateral sclerosis serum. Ann Neurol 40:695-700.

Athanassakis I, Protopapadakis E, Vassiliadis S (2000) Localization of pepstatin's inhibitory action during Fc-mediated antibody internalization: possible implications for antibody-mediated viral transmission. Cell Immunol 199:81-88.

Berridge MJ, Bootman MD, Roderick HL (2003) Calcium signalling: dynamics, homeostasis and remodelling. Nat Rev Mol Cell Biol 4:517-529.

Brooks BR, Shodis KA, Lewis DH, Rawling JD, Sanjak M, Belden DS, Hakim H, DeTan Y, Gaffney JM (1995) Natural history of amyotrophic lateral sclerosis. Quantification of symptoms, signs, strength, and function. Adv Neurol 68:163-184.

Bullens RW, O'Hanlon GM, Wagner E, Molenaar PC, Furukawa K, Plomp JJ, Willison HJ (2002) Complex gangliosides at the neuromuscular junction are membrane receptors for autoantibodies and botulinum neurotoxin but redundant for normal synaptic function. J Neurosci 22:6876-6884.

Chio A, Benzi G, Dossena M, Mutani R, Mora G (2005) Severely increased risk of amyotrophic lateral sclerosis among Italian professional football players. Brain 128:472-476.

Cleveland DW, Rothstein JD (2001) From Charcot to Lou Gehrig: deciphering selective motor neuron death in ALS. Nat Rev Neurosci 2:806-819.

Cui LY, Liu MS, Tang XF (2004) Single fiber electromyography in 78 patients with amyotrophic lateral sclerosis. Chin Med J (Engl) 117:1830-1833.

David G, Barrett EF (2003) Mitochondrial $\mathrm{Ca}^{2+}$ uptake prevents desynchronization of quantal release and minimizes depletion during repetitive stimulation of mouse motor nerve terminals. J Physiol (Lond) 548:425-438.

Day NC, Wood SJ, Ince PG, Volsen SG, Smith W, Slater CR, Shaw PJ (1997) Differential localization of voltage-dependent calcium channel $\alpha 1$ subunits at the human and rat neuromuscular junction. J Neurosci 17:6226-6235.

Delbono O, Garcia J, Appel SH, Stefani E (1991) Calcium current and charge movement of mammalian muscle: action of amyotrophic lateral sclerosis immunoglobulins. J Physiol (Lond) 444:723-742.

Demestre M, Pullen A, Orrell RW, Orth M (2005) ALS-IgG-induced selective motor neurone apoptosis in rat mixed primary spinal cord cultures. J Neurochem 94:268-275.

Doughty SW, Blaney FE, Orlek BS, Richards WG (1998) A molecular mechanism for toxin block in N-type calcium channels. Protein Eng 11:95-99.

Drachman DB (2000) Does autoimmunity play a role in amyotrophic lateral sclerosis? In: Amyotrophic lateral sclerosis (Brown RH, Meininger V, Swash M, eds), pp 327-339. London: Dunitz.

Engelhardt JI, Siklos L, Komuves L, Smith RG, Appel SH (1995) Antibodies to calcium channels from ALS patients passively transferred to mice selectively increase intracellular calcium and induce ultrastructural changes in motoneurons. Synapse 20:185-199.

Engelhardt JI, Siklos L, Appel SH (1997) Altered calcium homeostasis and ultrastructure in motoneurons of mice caused by passively transferred anti-motoneuronal IgG. J Neuropathol Exp Neurol 56:21-39.

Fratantoni SA, Weisz G, Pardal AM, Reisin RC, Uchitel OD (2000) Amyotrophic lateral sclerosis IgG-treated neuromuscular junctions develop sensitivity to L-type calcium channel blocker. Muscle Nerve 23:543-550.

Guegan C, Vila M, Rosoklija G, Hays AP, Przedborski S (2001) Recruitment of the mitochondrial-dependent apoptotic pathway in amyotrophic lateral sclerosis. J Neurosci 21:6569-6576.

Hua SY, Raciborska DA, Trimble WS, Charlton MP (1998) Different VAMP/synaptobrevin complexes for spontaneous and evoked transmitter release at the crayfish neuromuscular junction. J Neurophysiol 80:3233-3246.

Jayaraman T, Marks AR (1997) T cells deficient in inositol 1,4,5trisphosphate receptor are resistant to apoptosis. Mol Cell Biol 17:3005-3012.

Katz E, Protti DA, Ferro PA, Rosato Siri MD, Uchitel OD (1997) Effects of $\mathrm{Ca}^{2+}$ channel blocker neurotoxins on transmitter release and presynaptic currents at the mouse neuromuscular junction. Br J Pharmacol 121:1531-1540.

Kimura F, Smith RG, Delbono O, Nyormoi O, Schneider T, Nastainczyk W, Hofmann F, Stefani E, Appel SH (1994) Amyotrophic lateral sclerosis patient antibodies label $\mathrm{Ca}^{2+}$ channel alpha 1 subunit. Ann Neurol 35:164-171.

Llinas R, Sugimori M, Cherksey BD, Smith RG, Delbono O, Stefani E, Appel S (1993) IgG from amyotrophic lateral sclerosis patients increases current through P-type calcium channels in mammalian cerebellar Purkinje cells and in isolated channel protein in lipid bilayer. Proc Natl Acad Sci USA 90:11743-11747.

Maselli RA, Wollman RL, Leung C, Distad B, Palombi S, Richman DP, Salazar-Grueso EF, Roos RP (1993) Neuromuscular transmission in amyotrophic lateral sclerosis. Muscle Nerve 16:1193-1203.

McEnery MW, Snowman AM, Snyder SH (1994) The association of endogenous Go alpha with the purified omega-conotoxin GVIA receptor. J Biol Chem 269:5-8.

Medesan C, Cianga P, Mummert M, Stanescu D, Ghetie V, Ward ES (1998) Comparative studies of rat IgG to further delineate the Fc:FcRn interaction site. Eur J Immunol 28:2092-2100.

Mochida S, Sheng ZH, Baker C, Kobayashi H, Catterall WA (1996) Inhibition of neurotransmission by peptides containing the synaptic protein interaction site of N-type $\mathrm{Ca}^{2+}$ channels. Neuron 17:781-788.

Mohamed HA, Mosier DR, Zou LL, Siklos L, Alexianu ME, Engelhardt JI, Beers DR, Le WD, Appel SH (2002) Immunoglobulin Fc gamma receptor promotes immunoglobulin uptake, immunoglobulin-mediated calcium increase, and neurotransmitter release in motor neurons. J Neurosci Res 69:110-116.

Mosier DR, Baldelli P, Delbono O, Smith RG, Alexianu ME, Appel SH, Stefani E (1995) Amyotrophic lateral sclerosis immunoglobulins increase $\mathrm{Ca}^{2+}$ currents in a motoneuron cell line. Ann Neurol 37:102-109.

Mosier DR, Siklos L, Appel SH (2000) Resistance of extraocular motoneuron terminals to effects of amyotrophic lateral sclerosis sera. Neurology 54:252-255.

Muchnik S, Losavio A, Lorenzo SD (2002) Effect of amyotrophic lateral sclerosis serum on calcium channels related to spontaneous acetylcholine release. Clin Neurophysiol. 113:1066-1071.

Narita K, Akita T, Hachisuka J, Huang S, Ochi K, Kuba K (2000) Functional coupling of $\mathrm{Ca}^{2+}$ channels to ryanodine receptors at presynaptic terminals. Amplification of exocytosis and plasticity. J Gen Physiol 115:519-532.

Norris Jr FH, Gasteiger EL (1955) Action potentials of single motor units in normal muscle. Electroencephalogr Clin Neurophysiol Suppl 7:115-125.

Nyormoi O (1996) Proteolytic activity in amyotrophic lateral sclerosis IgG preparations. Ann Neurol 40:701-706.

Ober RJ, Radu CG, Ghetie V, Ward ES (2001) Differences in promiscuity for antibody-FcRn interactions across species: implications for therapeutic antibodies. Int Immunol 13:1551-1559.

Offen D, Halevi S, Orion D, Mosberg R, Stern-Goldberg H, Melamed E, Atlas D (1998) Antibodies from ALS patients inhibit dopamine release mediated by L-type calcium channels. Neurology 51:1100-1103.

Ordonez G, Sotelo J (1989) Antibodies against fetal muscle proteins in serum from patients with amyotrophic lateral sclerosis. Neurology 39:683-686.

Orrenius S, Zhivotovsky B, Nicotera P (2003) Regulation of cell death: the calcium-apoptosis link. Nat Rev Mol Cell Biol 4:552-565.

O’Shaughnessy TJ, Yan H, Kim J, Middlekauff EH, Lee KW, Phillips LH, Kim YI (1998) Amyotrophic lateral sclerosis: serum factors enhance spontaneous and evoked transmitter release at the neuromuscular junction. Muscle Nerve 21:81-90.

Otsu Y, Murphy TH (2004) Optical postsynaptic measurement of vesicle release rates for hippocampal synapses undergoing asynchronous release during train stimulation. J Neurosci 24:9076-9086.

Pagani R, Song M, McEnery M, Qin N, Tsien RW, Toro L, Stefani E, Uchitel OD (2004) Differential expression of alpha 1 and beta subunits of voltage dependent $\mathrm{Ca}^{2+}$ channel at the neuromuscular junction of normal and P/Q Ca ${ }^{2+}$ channel knockout mouse. Neuroscience 123:75-85.

Protti DA, Szczupak L, Scornik FS, Uchitel OD (1991) Effect of omegaconotoxin GVIA on neurotransmitter release at the mouse neuromuscular junction. Brain Res 557:336-339.

Pullen AH, Humphreys P (2000) Ultrastructural analysis of spinal mo- 
toneurones from mice treated with IgG from ALS patients, healthy individuals, or disease controls. J Neurol Sci 180:35-45.

Pullen AH, Demestre M, Howard RS, Orrell RW (2004) Passive transfer of purified IgG from patients with amyotrophic lateral sclerosis to mice results in degeneration of motor neurons accompanied by $\mathrm{Ca}^{2+}$ enhancement. Acta Neuropathol (Berl) 107:35-46.

Rizzoli SO, Betz WJ (2002) Effects of 2-(4-morpholinyl)-8-phenyl-4 H-1benzopyran-4-one on synaptic vesicle cycling at the frog neuromuscular junction. J Neurosci 22:10680-10689.

Rizzuto R, Brini M, Murgia M, Pozzan T (1993) Microdomains with high $\mathrm{Ca}^{2+}$ close to IP3-sensitive channels that are sensed by neighboring mitochondria. Science 262:744-747.

Robidoux J, Simoneau L, St-Pierre S, Ech-Chadli H, Lafond J (1998) Human syncytiotrophoblast NPY receptors are located on BBM and activate PLC-to-PKC axis. Am J Physiol 274:E502-E509.

Sara Y, Virmani T, Deak F, Liu X, Kavalali ET (2005) An isolated pool of vesicles recycles at rest and drives spontaneous neurotransmission. Neuron 45:563-573.

Schiavo G, Gu QM, Prestwich GD, Sollner TH, Rothman JE (1996) Calcium-dependent switching of the specificity of phosphoinositide binding to synaptotagmin. Proc Natl Acad Sci USA 93:13327-13332.

Siklos L, Engelhardt J, Harati Y, Smith RG, Joo F, Appel SH (1996) Ultrastructural evidence for altered calcium in motor nerve terminals in amyotropic lateral sclerosis. Ann Neurol 39:203-216.

Smith RG, Hamilton S, Hofmann F, Schneider T, Nastainczyk W, Birnbaumer L, Stefani E, Appel SH (1992) Serum antibodies to L-type calcium channels in patients with amyotrophic lateral sclerosis. $\mathrm{N}$ Engl J Med 327:1721-1728.

Smith RG, Alexianu ME, Crawford G, Nyormoi O, Stefani E, Appel SH (1994) Cytotoxicity of immunoglobulins from amyotrophic lateral sclerosis patients on a hybrid motoneuron cell line. Proc Natl Acad Sci USA 91:3393-3397.
Smith SA, Miller RG, Murphy JR, Ringel SP (1994) Treatment of ALS with high dose pulse cyclophosphamide. J Neurol Sci [Suppl] 124:84-87.

Stoop R, Poo MM (1995) Potentiation of transmitter release by ciliary neurotrophic factor requires somatic signaling. Science 267:695-699.

Strong MJ (2003) The basic aspects of therapeutics in amyotrophic lateral sclerosis. Pharmacol Ther 98:379-414.

Thomas MM, Dunn SM (1997) Amyotrophic lateral sclerosis immunoglobulins are ineffective in altering calcium influx through presynaptic voltage-sensitive calcium channels. Neurol Res 19:129-134.

Troost D, Van den Oord JJ, Vianney de Jong JM (1990) Immunohistochemical characterization of the inflammatory infiltrate in amyotrophic lateral sclerosis. Neuropathol Appl Neurobiol 16:401-410.

Uchitel OD, Appel SH, Crawford F, Sczcupak L (1988) Immunoglobulins from amyotrophic lateral sclerosis patients enhance spontaneous transmitter release from motor-nerve terminals. Proc Natl Acad Sci USA 85:7371-7374.

Uchitel OD, Scornik F, Protti DA, Fumberg CG, Alvarez V, Appel SH (1992) Long-term neuromuscular dysfunction produced by passive transfer of amyotrophic lateral sclerosis immunoglobulins. Neurology 42:2175-2180.

Vincent A (1999) Immunology of the neuromuscular junction and presynaptic nerve terminal. Curr Opin Neurol 12:545-551.

Yan HD, Lim W, Lee KW, Kim J (1997) Sera from amyotrophic lateral sclerosis patients reduce high-voltage activated $\mathrm{Ca}^{2+}$ currents in mice dorsal root ganglion neurons. Neurosci Lett 235:69-72.

Yang F, He X, Feng L, Mizuno K, Liu XW, Russell J, Xiong WC, Lu B (2001) PI-3 kinase and IP3 are both necessary and sufficient to mediate NT3induced synaptic potentiation. Nat Neurosci 4:19-28.

Zhainazarov AB, Annunziata P, Toneatto S, Cherubini E, Nistri A (1994) Serum fractions from amyotrophic lateral sclerosis patients depress voltage-activated $\mathrm{Ca}^{2+}$ currents of rat cerebellar granule cells in culture. Neurosci Lett 172:111-114. 